\title{
Simplicity from recoil: the three-loop soft function and factorization for the energy-energy correlation
}

\author{
Ian Moult ${ }^{a, b}$ and Hua Xing Zhu ${ }^{c}$ \\ ${ }^{a}$ Berkeley Center for Theoretical Physics, University of California, \\ Berkeley, CA 94720, U.S.A. \\ ${ }^{b}$ Theoretical Physics Group, Lawrence Berkeley National Laboratory, \\ Berkeley, CA 94720, U.S.A. \\ ${ }^{c}$ Zhejiang Institute of Modern Physics, Department of Physics, Zhejiang University, \\ Hangzhou, Zhejiang 310027, China \\ E-mail: ianmoult@lbl.gov, zhuhx@zju.edu.cn
}

ABSTRACT: We derive an operator based factorization theorem for the energy-energy correlation (EEC) observable in the back-to-back region, allowing the cross section to be written as a convolution of hard, jet and soft functions. We prove the equivalence of the soft functions for the EEC and color singlet transverse-momentum resummation to allloop order, and give their analytic result to three-loops. Large logarithms appearing in the perturbative expansion of the EEC can be resummed to all orders using renormalization group evolution. We give analytic results for all required anomalous dimensions to threeloop order, providing the first example of a transverse-momentum (recoil) sensitive $e^{+} e^{-}$ event shape whose anomalous dimensions are known at this order. The EEC can now be computed to next-to-next-to-next-to-leading logarithm matched to next-to-next-to-leading order, making it a prime candidate for precision QCD studies and extractions of the strong coupling constant. We anticipate that our factorization theorem will also be crucial for understanding non-perturbative power corrections for the EEC, and their relationship to those appearing in other observables.

KeYwords: Jets, NLO Computations

ARXIV EPRINT: 1801.02627 


\section{Contents}

1 Introduction 1

2 Kinematics 4

3 Factorization theorem for the EEC in the back-to-back region 6

3.1 Factorization theorem 6

$\begin{array}{ll}3.2 & \text { Renormalization group evolution } \\ & 12\end{array}$

4 Three-loop anomalous dimensions and soft function $\quad 15$

$\begin{array}{ll}4.1 \text { Anomalous dimensions } & 15\end{array}$

$\begin{array}{lll}4.2 & \text { Equivalence of soft functions and the three-loop boundary condition } & 17\end{array}$

$\begin{array}{llr}5 & \text { Resummation formula } & 20\end{array}$

6 Conclusions 21

A Matching coefficients for the TMDFF and EEC jet function 23

$\begin{array}{ll}\text { B Logarithmic structure to NNLO } & 25\end{array}$

\section{Introduction}

Event shapes in $e^{+} e^{-}$provide a theoretically and experimentally clean environment for precision studies of QCD and extractions of the strong coupling constant, $\alpha_{s}$. The perturbative description of event shapes requires both the calculation of fixed order corrections, which are currently known to next-to-next-to-leading order (NNLO) [1-4], as well as the all orders resummation of singular logarithmic terms [5]. There has been significant progress in both of these areas in recent years: advances in fixed order subtractions [6-9], have enabled a number of new NNLO calculations [6,7], and resummation to next-to-next-to-leading logarithmic (NNLL) order has been performed for a wide variety of observables [10-16], and implemented in a fully generic manner $[17,18]$.

Resummation to next-to-next-to-next-to-leading logarithmic $\left(\mathrm{N}^{3} \mathrm{LL}\right)$ accuracy has been achieved using the soft collinear effective theory (SCET) [19-22], which allows resummation to be performed using renormalization group evolution, in virtuality or rapidity [23-25], of field theoretic operators. Resummed predictions at $\mathrm{N}^{3} \mathrm{LL}$ accuracy have been made for thrust [26, 27], the $C$-parameter [28] and heavy jet mass [29], enabling precision extractions of $\alpha_{s}[26,27,29,30]$. However, all of these observables are "recoil free", or transverse-momentum $\left(q_{T}\right)$ insensitive (described by $\mathrm{SCET}_{\mathrm{I}}$ ), meaning that at leading power, soft partons are not able to recoil the $q_{T}$ of the jet. Unfortunately, there are no examples of $q_{T}$ sensitive observables in $e^{+} e^{-}$which are known at $\mathrm{N}^{3} \mathrm{LL}$ accuracy, which could complement $\alpha_{s}$ fits using recoil free observables. Recoil typically significantly 
complicates perturbative calculations. For example, calculations of jet broadening [31-33] are complicated by issues of recoil, jet regions, and by the fact that it is a scalar sum which become complicated at multiple emissions. Indeed, elliptic functions appear already at NNLL for broadening [11], making extensions to higher orders seem difficult.

Recently, the three-loop soft function governing the color singlet $q_{T}$ spectrum at small $q_{T}$ was computed [34]. This calculation used bootstrap techniques from $\mathcal{N}=4$ super YangMills theory [35-39], a supersymmetric decomposition in transcendental weight, a newly introduced rapidity regulator [40], multi-dimensional factorization [41, 42], and recently computed master integrals [43-48]. The final result has a remarkably simple structure and exhibits interesting relations to other anomalous dimensions $[34,49,50]$. The computation to this order was ultimately enabled by the simple structure of the observable: it is a vector sum, which preserves the maximal number of rotational symmetries, and does not involve any jet regions, or projections onto axes whose precise definition can modify the perturbative structure $[51,52]$. It is therefore interesting to ask whether this anomalous dimension controls the resummation of any $e^{+} e^{-}$event shape observables. This is interesting both phenomenologically, as it could provide information for $\alpha_{s}$ extractions complimentary to that from recoil free observables, as well as for understanding all orders relations between different observables.

In this paper, we derive an all orders factorization theorem for the energy-energy correlation (EEC) in the back-to-back region. In this factorization theorem, the soft radiation does not contribute directly to the observable at leading power, but instead contributes only via recoil. We are able to show that the soft function appearing in the factorization is identical to that for the color singlet $q_{T}$ distribution, up to the direction of the Wilson lines. ${ }^{1}$ Using a recently introduced rapidity regulator [40], which allows both the regulator and the measurement function to be described by spacetime shifts of the Wilson lines, we prove that the soft function is invariant under the crossing of the Wilson lines, allowing us to use the recently derived results for $q_{T}$ soft function to derive the anomalous dimension and soft function for the EEC. This provides the first example of a $q_{T}$ sensitive observable in $e^{+} e^{-}$ whose anomalous dimensions are known to three-loops. It also illustrates the utility of operator definitions in factorization theorems, which allow for the identification of universal structures in apparently different situations. As a further consequence of our analysis, the anomalous dimension and soft function could also be used for identified hadron production in the back-to-back limit, allowing it to be extended to $\mathrm{N}^{3} \mathrm{LL}$ perturbative accuracy.

The EEC, one of the earliest examples of an infrared and collinear (IRC) safe observable, is defined as [54]

$$
\mathrm{EEC}=\sum_{a, b} \int d \sigma_{V \rightarrow a+b+X} \frac{2 E_{a} E_{b}}{Q^{2} \sigma_{\mathrm{tot}}} \delta\left(\cos \left(\theta_{a b}\right)-\cos (\chi)\right)
$$

\footnotetext{
${ }^{1}$ The similarity between the resummation for EEC and $q_{T}$ in the back-to-back region has long been known, and has been used to perform the resummation to NNLL using the Collins-Soper-Sterman form as an ansatz, see e.g. $[10,16]$. However, we were not able to find a factorization theorem for the EEC in the literature, or an all orders proof of this relation between the anomalous dimensions. The steps for a proof of factorization along with a leading log resummation formula were given in [53].
} 


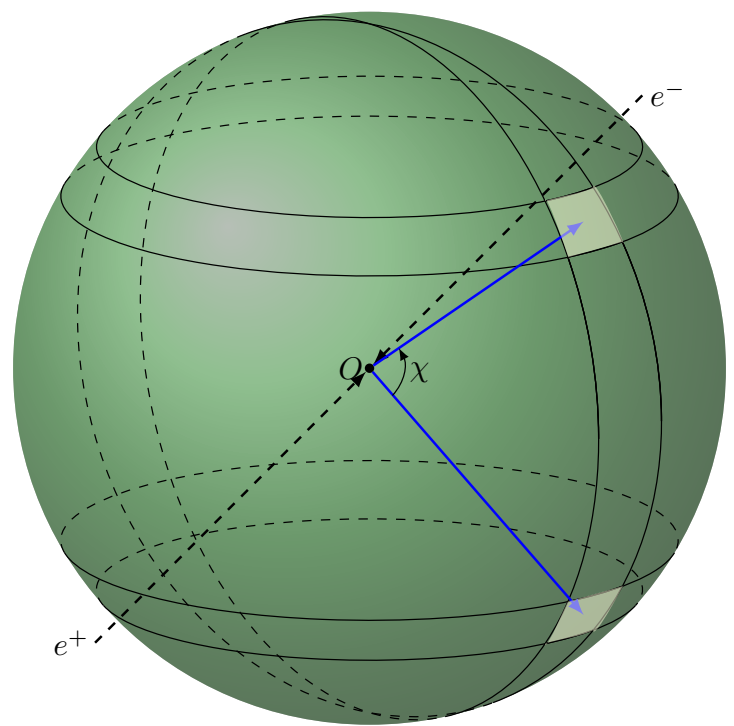

Figure 1. An illustration of the EEC observable in $e^{+} e^{-}$annihilation, which is defined as the energy-energy correlation of two calorimeter cells with opening angle $\chi$.

where the sum is over all different pairs of hadrons $h_{a}$ and $h_{b}$ in the event, cf. figure 1 . It has been studied extensively in the QCD literature [10, 55-66], and has been computed analytically to NLO in planar $\mathcal{N}=4$ super Yang-Mills theory, exploiting a relation to correlation functions [67-69], as well as at strong-coupling [70] using the AdS/CFT correspondence [71]. There has also been progress towards the NLO calculation in QCD [72]. Recently it was computed at NNLL+NNLO [16] using the NNLO calculation of $[6,7]$ and used to fit $\alpha_{s}$ from data. Our results will allow this to be extended to $\mathrm{N}^{3} \mathrm{LL}$ ' $+\mathrm{NNLO}$, matching the state of the art precision for recoil free $\left(\mathrm{SCET}_{\mathrm{I}}\right)$ observables. ${ }^{2}$

An outline of this paper is as follows. In section 2 we discuss the kinematics of the EEC in the back-to-back limit, and illustrate the relationship between the EEC and $q_{T}$. In section 3 we present our factorization theorem for the EEC observable, discussing in detail how soft radiation contributes to the observable. In section 4 we prove that both the anomalous dimensions, as well as the full soft function, are identical to those governing the color singlet $q_{T}$ spectrum, and we use this relation to give explicit results for the soft function to three-loop order. In section 5 we use our factorization theorem to give the all orders form for the resummed cross section in the back-to-back limit. We conclude and discuss future directions in section 6. Additional calculations, and a comparison to NNLO of the logarithmic structure as predicted by our factorization theorem with results in the literature, are given in the appendices.

\footnotetext{
${ }^{2}$ Here the prime on the logarithmic accuracy indicates the inclusion of the three-loop boundary conditions for the soft and collinear functions, as has been included for thrust and $C$-parameter [26-28]. See also [73] for a detailed discussion of order counting.
} 


\section{Kinematics}

In this section we discuss in detail the kinematics of the EEC observable in the back-toback region, $\chi \rightarrow \pi$ in eq. (1.1). This will be important in understanding the derivation of the factorization theorem, and the relation to the $q_{T}$ observable. It will be convenient to work with the dimensionless variable

$$
z=\frac{1-\cos \theta_{i j}}{2}
$$

in terms of which we have

$$
\frac{d \sigma}{d z}=\frac{1}{2} \sum_{i j} \int d x_{i} d x_{j} x_{i} x_{j} \frac{d^{3} \sigma}{d x_{i} d x_{j} d z},
$$

where $\frac{d^{3} \sigma}{d x_{i} d x_{j} d z}$ is the triple differential cross section measuring the energy fraction with respect to half of the center of mass energy, $x_{i, j}=2 E_{i, j} / Q$, and relative angle $z$. Note that this triple differential distribution is not IRC safe. IRC safety is recovered after summing over different particles. Here the summation is over different pair of hadron in the final state with momentum $p_{i}$ and $p_{j}$. The back-to-back limit is then characterized by $\chi=\theta_{i j} \rightarrow \pi$, or $z \rightarrow 1$.

In the back-to-back limit, the event consists of two nearly back-to-back jets, along with additional low energy (soft) radiation. Additional hard jets are power suppressed (for a detailed discussion, see [25]). This situation is illustrated schematically in figure 2. We denote the momentum of the two jets by $p_{a}^{\mu}=\left(p_{a}^{0}, \vec{p}_{a}\right)$ and $p_{b}^{\mu}=\left(p_{b}^{0}, \vec{p}_{b}\right)$. We then define two light-cone directions $n_{a}^{\mu}=\left(1, \vec{n}_{a}\right)$ and $n_{b}^{\mu}=\left(1, \vec{n}_{b}\right)$, with $\vec{n}_{a(b)}=\vec{p}_{a(b)} /\left|\vec{p}_{a(b)}\right|$. We also define the conjugate directions $\bar{n}_{a(b)}=\left(1,-\vec{n}_{a(b)}\right)$. The relevant modes in the effective theory are easily determined by considering on-shell modes that contribute to the EEC observable at leading power. They are found to be soft, collinear, and anti-collinear, with the scalings in light-cone coordinates

$$
p_{s} \sim Q(\lambda, \lambda, \lambda), \quad p_{c} \sim Q\left(\lambda^{2}, 1, \lambda\right), \quad p_{\bar{c}} \sim Q\left(1, \lambda^{2}, \lambda\right),
$$

where

$$
\lambda \sim \sqrt{1-z} .
$$

In particular, we see that the EEC is an $\mathrm{SCET}_{\mathrm{II}}$ [74] observable. This is intuitively obvious, since the EEC directly measures angles between hadrons and is therefore sensitive to recoil at leading power. With the above definition, we have $\bar{n}_{a(b)} \cdot p_{a(b)}=Q+\mathcal{O}(\lambda Q)$.

Using the definition of the observable in eq. (2.2), and the power counting of the modes in eq. (2.3), we can now expand the EEC observable to leading power in the $z \rightarrow 1$ limit. We begin by noting that the contribution of soft modes to the observable is power suppressed. Soft radiation therefore will contribute only by recoiling the jet sectors. This is quite interesting, and in particular, it implies that it is sufficient to know the total vector transverse momentum of the soft sector. ${ }^{3}$

\footnotetext{
${ }^{3}$ We find it interesting that recoil, which often leads to complications, in fact leads to the remarkable simplicity of the soft function for the EEC.
} 


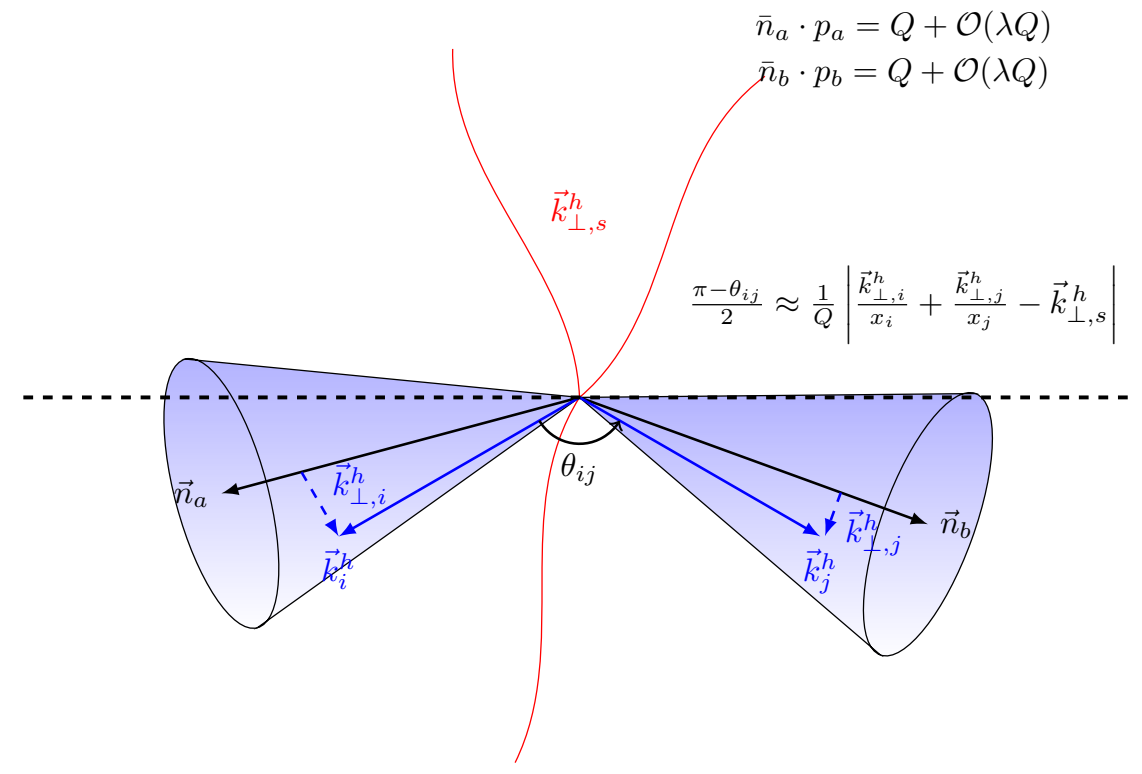

Figure 2. A schematic of the kinematics relevant for the description of the EEC observable in the back-to-back limit, $z \rightarrow 1$. The total transverse momentum of final-state hadrons perpendicular to the thrust axis (the black dashed line) is denoted as $\vec{k}_{\perp, s}^{h}$. The three-momentum of a pair of collinear hadrons which enter weighted sum in eq. (2.2) are denoted as $\vec{k}_{i, j}^{h}$. The corresponding transverse components perpendicular to the jet axis $\vec{n}_{a, b}$ are $\vec{k}_{\perp, i}^{h}$ and $\vec{k}_{\perp, j}^{h}$. Soft radiation acts only to recoil the two collinear sectors, denoted by $\vec{n}_{a, b}$, with respect to each other, but does not contribute directly to the observable.

Contributions to the observable arise only from correlations between collinear partons in different collinear sectors. It is therefore a simple geometric exercise to relate their perpendicular momentum to $z$, as relevant for the EEC. Considering the configuration shown in figure 2 , to leading power we find

$$
1-z=\frac{1}{Q^{2}}\left|\frac{\vec{k}_{\perp, i}^{h}}{x_{i}}+\frac{\vec{k}_{\perp, j}^{h}}{x_{j}}-\vec{k}_{\perp, s}^{h}\right|^{2}+\mathcal{O}(1-z),
$$

where $k_{\perp, s}^{h}$ is the total transverse momentum of soft final-state hadrons relative to thrust axis, and $k_{\perp, i(j)}^{h}$ is the tranverse momentum of a collinear hadron relative to its respective jet axis, defined as the direction with largest energy flow. We emphasize that eq. (2.5) only holds in the back-to-back limit. With these definitions, we have the conservation of transverse momentum within each jet

$$
\sum_{i \in \text { jet a }} \vec{k}_{\perp, i}^{h}=\mathcal{O}\left(\lambda^{2} Q\right), \quad \sum_{j \in \text { jet b }} \vec{k}_{\perp, j}^{h}=\mathcal{O}\left(\lambda^{2} Q\right)
$$

and the conservation of total transverse momentum perpendicular to the thrust axis

$$
\vec{k}_{\perp, s}^{h}+\vec{p}_{\perp, a}+\vec{p}_{\perp, b}=\mathcal{O}\left(\lambda^{2} Q\right)
$$


where $\vec{p}_{\perp, a(b)}$ is the transverse momentum of jet a(b) perpendicular to the thrust axis. Eq. (2.5) clearly shows the relationship between the EEC observable and $q_{T}$. This expression also hints at the simplicity of the EEC. Most importantly, $1-z$ is related to the vector sum of the transverse-momentum in the different sectors, and in particular, the only property of the soft radiation that is measured is the total transverse-momentum. This is much simpler than other recoil sensitive $e^{+} e^{-}$observables such as broadening, where it is ultimately the scalar sum of the transverse momentum that is measured, making the measurement function extremely complicated for configurations with multiple emissions.

\section{Factorization theorem for the EEC in the back-to-back region}

Having understood the kinematics of the EEC observable in the back-to-back region, we can now derive a factorization theorem describing the all orders singular behavior in the $z \rightarrow 1(\chi \rightarrow \pi)$ limit. This factorization theorem will allow us to express the leading power cross section for the EEC as a product (convolution) of a hard matching coefficient, $H$, a soft function, $S$, which describes wide angle soft radiation, and jet functions, $J$, which describe collinear radiation in the jets. Each of these functions will describe the dynamics at a single scale, and large logarithms in the cross section can be resummed through their renormalization group evolution, which is given in section 3.2.

We will present this factorization in the language of SCET [19-22], giving gauge invariant operator definitions for the jet and soft functions. While the resummation of the EEC to NNLL order has been performed in the Collins-Soper-Sterman (CSS) [53, 75-78] formalism (see e.g. $[10,16]$ ), we are not aware of a factorization theorem in terms of hard, jet and soft functions. Such a factorization will ultimately allow us to prove the equivalence of the soft function for the EEC and for $q_{T}$ in color singlet production, and to extend the perturbative accuracy of the EEC observable to $\mathrm{N}^{3} \mathrm{LL}$.

\subsection{Factorization theorem}

We will derive the factorization theorem for the case of $e^{+} e^{-} \rightarrow$ dijets through an off-shell photon or $Z$. The extension to other underlying hard processes, such as $e^{+} e^{-} \rightarrow g g$ through an off-shell Higgs, is trivial. Our proof will use as a starting point the factorization theorem for identified hadron production in the back-to-back limit [53, 75], and we will formulate the factorization for the EEC observable by marginalizing over this factorization theorem. Since a factorization theorem, and in particular the cancellation of Glauber modes, has been proven for back-to-back identified hadron production, we will also be able to use this argument to conclude that Glauber modes do not contribute to the EEC and therefore that they do not violate the factorization at all orders.

Since the derivation of the factorization starts from identified hadron production in the back-to-back limits, we will use fragmentation functions, and their transverse momentum dependent (TMD) counterparts, extensively. We therefore begin by reviewing their 
definitions. We define the standard fragmentation functions (FFs) as [79-82]

$$
\begin{aligned}
f_{q \rightarrow h}\left(z_{h}\right)=\frac{1}{4 z_{h} N_{c}} \sum_{X} \int \frac{d \xi^{+}}{4 \pi} e^{-i p_{h}^{-} \xi^{+} / z_{h}} & \\
& \left\langle 0\left|T\left[\tilde{W}_{n}^{\dagger} q_{j}\right]_{a}\left(\frac{\xi^{+}}{2}\right)\right| X, h\right\rangle \gamma_{i j}^{-}\left\langle X, h\left|\bar{T}\left[\bar{q}_{i} \tilde{W}_{n}\right]_{a}\left(-\frac{\xi^{+}}{2}\right)\right| 0\right\rangle,
\end{aligned}
$$

and their TMD extensions (TMDFFs) as $[83,84]$

$$
\begin{aligned}
& F_{q \rightarrow h}\left(\vec{b}_{\perp}, z_{h}\right)=\frac{1}{4 z_{h} N_{c}} \sum_{X} \int \frac{d \xi^{+}}{4 \pi} e^{-i p_{h}^{-} \xi^{+} / z_{h}} \\
&\left\langle 0\left|T\left[\tilde{W}_{n}^{\dagger} q_{j}\right]_{a}\left(\frac{\xi}{2}\right)\right| X, h\right\rangle \gamma_{i j}^{-}\left\langle X, h\left|\bar{T}\left[\bar{q}_{i} \tilde{W}_{n}\right]_{a}\left(-\frac{\xi}{2}\right)\right| 0\right\rangle .
\end{aligned}
$$

Here $W_{n}$ is a Wilson line, defined in momentum space as

$$
W_{n}=\left[\sum_{\text {perms }} \exp \left(-\frac{g}{\bar{n} \cdot \mathcal{P}} \bar{n} \cdot A_{n}(x)\right)\right],
$$

and $q$ are lightcone projected fermionic fields. Here $\xi=\left(\xi^{+}, 0^{-}, \vec{b}_{\perp}\right)$, with $\vec{b}_{\perp}$ the conjugate variable to $\vec{k}_{\perp}^{h}$, the transverse momentum of $h$ perpendicular to jet axis $\vec{n}$, which is aligned with the total jet three momentum. The lightcone vector $n^{\mu}$ in the operator definition of $f_{q \rightarrow h}$ and $F_{q \rightarrow h}$ is then defined by $n^{\mu}=(1, \vec{n})$. We also define the conjugate lightcone vector $\bar{n}^{\mu}=(1,-\vec{n})$. We are therefore working in the center-of-mass frame, in contrast to the more conventional hadron frame [83]. Note that the only difference between the definition for the TMDFF and the standard FF is in the positions of the fields. The renormalized fragmentation functions satisfy the following sum rule

$$
\sum_{h} \int_{0}^{1} d x x f_{q \rightarrow h}\left(x, \mu_{F}\right)=1,
$$

which will play an important role in our derivation.

To derive a factorization theorem for the EEC in the back-to-back region, we begin by factorizing the multi-differential cross section which appears in its definition

$$
\frac{d \sigma}{d z}=\frac{1}{2} \sum_{i j} \int d x_{i} d x_{j} x_{i} x_{j} \frac{d^{3} \sigma}{d x_{i} d x_{j} d z} .
$$

Furthermore, in the back-to-back limit, we can exchange the variable $z$ for an auxiliary transverse momentum, $\vec{k}_{\perp}=\vec{k}_{\perp, i}^{h} / x_{i}+\vec{k}_{\perp, j}^{h} / x_{j}-\vec{k}_{\perp, s}^{h}$, by writing

$$
\frac{d^{3} \sigma}{d x_{i} d x_{j} d z}=\int d^{2} \vec{k}_{\perp} \frac{d^{3} \sigma}{d x_{i} d x_{j} d^{2} \vec{k}_{\perp}} \delta\left(1-z-\frac{\vec{k}_{\perp}^{2}}{Q^{2}}\right) .
$$

In the back-to-back limit we can write a factorized expression for this cross section using the result for identified hadrons in the back-to-back region as was studied in the seminal 
papers $[53,75]$. In $[53,75]$ a factorization theorem was proven, and in particular it was shown that Glauber modes do not contribute, using techniques developed in [85-87] (see also $[82,83]$ for a review). Since we will formulate our factorization for the EEC from this starting point, this implies also that Glaubers cancel from the EEC observable. Using these results, we have

$$
\begin{aligned}
\frac{d^{3} \sigma}{d x_{i} d x_{j} d^{2} \vec{k}_{\perp}}= & H(Q, \mu) \int d^{2} \vec{k}_{\perp, i}^{h} \int d^{2} \vec{k}_{\perp, j}^{h} \int d^{2} \vec{k}_{\perp, s} \delta^{(2)}\left(\vec{k}_{\perp}-\left(\frac{\vec{k}_{\perp, i}^{h}}{x_{i}}+\frac{\vec{k}_{\perp, j}^{h}}{x_{j}}-\vec{k}_{\perp, s}\right)\right) \\
& \cdot F_{q \rightarrow i}\left(\vec{k}_{\perp, i}^{h}, x_{i}, \mu, \nu\right) F_{q \rightarrow j}\left(\vec{k}_{\perp, j}^{h}, x_{j}, \mu, \nu\right) S_{\mathrm{EEC}}\left(\vec{k}_{\perp, s}, \mu, \nu\right),
\end{aligned}
$$

where the triple differential distribution is written as convolution over transverse momentum of collinear hadrons perpendicular to jet axis, and transverse momentum of soft hadrons perpendicular to thrust axis. For the soft sector we do not distinguish partonic and hadronic momentum, as the impact of soft modes to the factorization formula is only through recoil. Here $H(Q, \mu)$ is the hard matching coefficient for $e^{+} e^{-} \rightarrow q \bar{q}, F_{q \rightarrow i}$ are the transverse momentum dependent fragmentation functions defined in eq. (3.2), and $S_{\text {EEC }}$ is the soft function. The $\mu$ and $\nu$ are the virtuality and rapidity renormalization scales, respectively. The dependence on rapidity scale arises because the naive TMD fragmentation function and soft function suffer from rapidity divergences, and need regularization and renormalization, similar to the TMD PDF. The renormalization group evolution of each of the functions appearing in the factorization theorem will be given in section 3.2.

The soft function, $S_{\mathrm{EEC}}$, is defined as a vacuum matrix element of Wilson lines. Since it will play a central role in our discussion, we will carefully define $S_{\mathrm{EEC}}$, paying particular attention to the directions of the Wilson lines, and the definition of the rapidity regulator. We begin by defining four distinct soft Wilson lines

$$
\begin{aligned}
& S_{n+}(z)=P \exp \left[i g \int_{-\infty}^{0} d s n \cdot A_{u s}(z+s n)\right], \\
& S_{n-}^{\dagger}(z)=\bar{P} \exp \left[-i g \int_{-\infty}^{0} d s n \cdot A_{u s}(z+s n)\right], \\
& S_{n+}^{\dagger}(z)=P \exp \left[i g \int_{0}^{\infty} d s n \cdot A_{u s}(z+s n)\right], \\
& S_{n-}(z)=\bar{P} \exp \left[-i g \int_{0}^{\infty} d s n \cdot A_{u s}(z+s n)\right] .
\end{aligned}
$$

Here $z^{\mu}$ is a reference vector defining the starting (ending) position of the Wilson line. For a detailed discussion of the Wilson line directions appearing in soft functions arising from factorization, see e.g. [88, 89]. The Wilson lines in different directions will be required to discuss both the soft functions appearing for the EEC, and for the color singlet $q_{T}$ spectrum, and to allow for an understanding of the relation between the soft functions appearing in these two cases. 
The soft function requires a rapidity regulator to be well defined. Here we use the recently introduced rapidity regulator of [40], which is implemented by displacing the Wilson lines from the origin. This is most easily formulated in position space (impact parameter space), obtained by performing a Fourier transform in the $\perp$ momentum [75]. Here we take $\vec{b}_{\perp}$ to be conjugate to $\vec{k}_{\perp}$. The offset of the Wilson lines is defined as

$$
y_{\nu}\left(\vec{b}_{\perp}\right)=\left(i b_{0} / \nu, i b_{0} / \nu, \vec{b}_{\perp}\right)
$$

where $b_{0}=2 e^{-\gamma_{E}}$. We can now define the soft function for the EEC as

$$
S_{\mathrm{EEC}}\left(\vec{b}_{\perp}, \mu, \nu\right)=\lim _{\nu \rightarrow+\infty} \frac{1}{N_{c}} \operatorname{tr}\left\langle 0\left|T\left[S_{\bar{n}+}^{\dagger}(0) S_{n-}(0)\right] \bar{T}\left[S_{n+}^{\dagger}\left(y_{\nu}\left(\vec{b}_{\perp}\right)\right) S_{\bar{n}-}\left(y_{\nu}\left(\vec{b}_{\perp}\right)\right)\right]\right| 0\right\rangle .
$$

The limit appearing in eq. (3.13) means to keep only the leading terms, dropping all terms that vanish as $\nu \rightarrow \infty$. The shift of the Wilson lines implements both the measurement of the $\perp$ momentum, as well as the regularization. Since the regularization can be implemented as a spacetime shift, it is well defined non-perturbatively. For a detailed discussion of the properties of this regulator, see [40].

The full expression for the leading power EEC cross section in the back-to-back limit can now be written as

$$
\begin{aligned}
\frac{d \sigma}{d z}= & \frac{1}{2} \sum_{i j} \int d x_{i} d x_{j} x_{i} x_{j} \int d^{2} \vec{k}_{\perp} \delta\left(1-z-\frac{\vec{k}_{\perp}^{2}}{Q^{2}}\right) \\
& \cdot H(Q, \mu) \int d^{2} \vec{k}_{\perp, i}^{h} \int d^{2} \vec{k}_{\perp, j}^{h} \int d^{2} \vec{k}_{\perp, s} \delta^{(2)}\left(\vec{k}_{\perp}-\left(\frac{\vec{k}_{\perp, i}^{h}}{x_{i}}+\frac{\vec{k}_{\perp, j}^{h}}{x_{j}}-\vec{k}_{\perp, s}\right)\right) \\
& \cdot F_{q \rightarrow i}\left(\vec{k}_{\perp, i}^{h}, x_{i}, \mu, \nu\right) F_{q \rightarrow j}\left(\vec{k}_{\perp, j}^{h}, x_{j}, \mu, \nu\right) S_{\mathrm{EEC}}\left(\vec{k}_{\perp, s}, \mu, \nu\right) .
\end{aligned}
$$

In its current form, this expression is still quite complicated, and furthermore, it involves the non-perturbative TMDFFs, despite the fact that the EEC is an IRC safe observable.

To simplify this result, we can perform an operator product expansion (OPE) of the TMDFF onto the standard FF, and use the sum rule of eq. (3.4) to eliminate the dependence on the FF. The OPE of the TMD FF onto the standard FF is given in momentum space by $[83,90-92]$

$$
F_{i \rightarrow h}\left(\vec{k}_{\perp}^{h}, z_{h}\right)=\sum_{j} \int \frac{d z}{z^{3}} \mathcal{I}_{i j}\left(\frac{\vec{k}_{\perp}^{h}}{z}, \frac{z_{h}}{z}\right) f_{j \rightarrow h}(z, \mu)\left[1+\mathcal{O}\left(\frac{\Lambda_{\mathrm{QCD}}^{2}}{\left(\vec{k}_{\perp}^{h}\right)^{2}}\right)\right]
$$

Here $\mathcal{I}_{i j}$ are infrared finite matching coefficients. Explicit results are given in appendix A. Inserting this expression into eq. (3.24), and changing variables to

$$
\tau_{i}=\frac{x_{i}}{z_{i}}, \quad d x_{i} d z_{i}=z_{i} d \tau_{i} d z_{i}
$$


We then find

$$
\begin{aligned}
\frac{d \sigma}{d z}= & \frac{1}{2} \sum_{i j} \int d \tau_{i} d \tau_{j} \tau_{i} \tau_{j} \int d^{2} \vec{k}_{\perp} \delta\left(1-z-\frac{\vec{k}_{\perp}^{2}}{Q^{2}}\right) \\
& \cdot H(Q) \int d^{2} \vec{k}_{\perp, i} \int d^{2} \vec{k}_{\perp, j} \int d^{2} \vec{k}_{\perp, s} \delta^{(2)}\left(\vec{k}_{\perp}-\left(\frac{\vec{k}_{\perp, i}}{\tau_{i}}+\frac{\vec{k}_{\perp, j}}{\tau_{j}}-\vec{k}_{\perp, s}\right)\right) \\
& \cdot \mathcal{I}_{q i}\left(\vec{k}_{\perp, i}, \tau_{i}\right)\left[\sum_{h} \int d z_{i} z_{i} f_{i \rightarrow h}\left(z_{i}, \mu\right)\right] \cdot \mathcal{I}_{q j}\left(\vec{k}_{\perp, j}, \tau_{j}\right)\left[\sum_{h^{\prime}} \int d z_{j} z_{j} f_{j \rightarrow h^{\prime}}\left(z_{j}, \mu\right)\right] \\
& \cdot S_{\mathrm{EEC}}\left(\vec{k}_{\perp, s}\right),
\end{aligned}
$$

where we have changed the convolution from hadronic transverse momentum $\vec{k}_{\perp, i(j)}^{h}$ to partonic transverse momentum $\vec{k}_{\perp, i(j)}$. The relation between hadronic and partonic transverse momentum is given by $\vec{k}_{\perp, i}^{h}=z_{i} \vec{k}_{\perp, i}$ and $\vec{k}_{\perp, j}^{h}=z_{j} \vec{k}_{\perp, j}$, which hold up to $\mathcal{O}\left(\Lambda_{\mathrm{QCD}}\right)$. We also use this relation to rewrite the measurement function. It then allows us to use the momentum-conservation sum rule

$$
\sum_{h} \int d z z f_{j \rightarrow h}(z, \mu)=1
$$

to cancel non-perturbative fragmentation functions, and we have

$$
\begin{aligned}
\frac{d \sigma}{d z}= & \frac{1}{2} \sum_{i j} \int d \tau_{i} d \tau_{j} \tau_{i} \tau_{j} \int d^{2} \vec{k}_{\perp} \delta\left(1-z-\frac{\vec{k}_{\perp}^{2}}{Q^{2}}\right) \\
& \cdot H(Q) \int d^{2} \vec{k}_{\perp, i} \int d^{2} \vec{k}_{\perp, j} \int d^{2} \vec{k}_{\perp, s} \delta^{(2)}\left(\vec{k}_{\perp}-\left(\frac{\vec{k}_{\perp, i}}{\tau_{i}}+\frac{\vec{k}_{\perp, j}}{\tau_{j}}-\vec{k}_{\perp, s}\right)\right) \\
& \cdot \mathcal{I}_{q i}\left(\vec{k}_{\perp, i}, \tau_{i}\right) \cdot \mathcal{I}_{q j}\left(\vec{k}_{\perp, j}, \tau_{j}\right) S_{\mathrm{EEC}}\left(\vec{k}_{\perp, s}\right) .
\end{aligned}
$$

This makes it clear that what we have is an expression in terms of the perturbative matching coefficients for the TMDFFs, $\mathcal{I}_{i j}$, which are by construction IR finite. This is of course not a surprise, since the EEC observable is IRC safe, however, it is interesting to see explicitly how it arises from the sum rule for the FFs.

We can further simplify the convolution structure by transforming to impact parameter space [75]. In addition to simplifying the convolution in the $k_{\perp, i}$ variables, as is familiar from the case of $q_{T}$ factorization, here we will find that this also simplifies the integrals over the momentum fractions $x_{i}$. Using the Fourier representation of delta function, we can write

$$
\delta^{(2)}\left(\vec{k}_{\perp}-\left(\frac{\vec{k}_{\perp, i}}{\tau_{i}}+\frac{\vec{k}_{\perp, j}}{\tau_{j}}-\vec{k}_{\perp, s}\right)\right)=\int \frac{d^{2} \vec{b}_{\perp}}{(2 \pi)^{2}} \exp \left[-i \vec{b}_{\perp} \cdot \vec{k}_{\perp}+i \vec{b}_{\perp} \cdot\left(\frac{\vec{k}_{\perp, i}}{\tau_{i}}+\frac{\vec{k}_{\perp, j}}{\tau_{j}}-\vec{k}_{\perp, s}\right)\right] .
$$


The momentum convolutions are now in complete factorized form. We define the Fouriertransformed matching coefficients and soft function as

$$
\begin{aligned}
& \mathcal{I}_{q i}\left(\vec{b}_{\perp, i}, x_{i}, \mu, \nu\right)=\int d^{2} \vec{k}_{\perp, i} \mathcal{I}_{q i}\left(\vec{k}_{\perp, i}, x_{i}, \mu, \nu\right) e^{i \vec{b}_{\perp, i} \cdot \vec{k}_{\perp, i}}, \\
& S_{\mathrm{EEC}}\left(\vec{b}_{\perp, s}, \mu, \nu\right)=\int d^{2} \vec{k}_{\perp, s} S_{\mathrm{EEC}}\left(\vec{k}_{\perp, s}, \mu, \nu\right) e^{i \vec{b}_{\perp, s} \cdot \vec{k}_{\perp, s}},
\end{aligned}
$$

where to simplify notation, we use only the argument of the function to indicate that it is Fourier transformed. This allows us to simplify our factorized expression to

$$
\begin{aligned}
\frac{d \sigma}{d z}= & \frac{1}{2} \sum_{i j} \int d x_{i} d x_{j} x_{i} x_{j} \int d^{2} \vec{k}_{\perp} \delta\left(1-z-\frac{\vec{k}_{\perp}^{2}}{Q^{2}}\right) \\
& \cdot H(Q) \int \frac{d^{2} \vec{b}_{\perp}}{(2 \pi)^{2}} e^{-i \vec{b}_{\perp} \cdot \vec{k}_{\perp}} \cdot \mathcal{I}_{q i}\left(\frac{\vec{b}_{\perp}}{x_{i}}, x_{i}, \mu, \nu\right) \mathcal{I}_{q j}\left(\frac{\vec{b}_{\perp}}{x_{j}}, x_{j}, \mu, \nu\right) S_{\mathrm{EEC}}\left(\vec{b}_{\perp}, \mu, \nu\right) \\
= & \int d^{2} \vec{k}_{\perp} \delta\left(1-z-\frac{\vec{k}_{\perp}^{2}}{Q^{2}}\right) \int \frac{d^{2} \vec{b}_{\perp}}{(2 \pi)^{2}} e^{-i \vec{b}_{\perp} \cdot \vec{k}_{\perp}} \cdot H(Q, \mu) \\
& \cdot\left[\sum_{i} \int d x_{i} x_{i} \mathcal{I}_{q i}\left(\frac{\vec{b}_{\perp}}{x_{i}}, x_{i}, \mu, \nu\right)\right]\left[\sum_{j} \int d x_{j} x_{j} \mathcal{I}_{q j}\left(\frac{\vec{b}_{\perp}}{x_{j}}, x_{j}, \mu, \nu\right)\right] S_{\mathrm{EEC}}\left(\vec{b}_{\perp}, \mu, \nu\right) .
\end{aligned}
$$

In this form, it is clear that only certain flavor summed moments of the matching coefficients for the fragmentation functions appear, and furthermore, that the integrals in the momentum fraction variables, $x_{i}$, are factorized. In particular, we can define the quark jet function relevant for the EEC as

$$
J_{\mathrm{EEC}}^{q}\left(\vec{b}_{\perp}\right)=\sum_{i} \int_{0}^{1} d x x \mathcal{I}_{q i}\left(\frac{\vec{b}_{\perp}}{x}, x\right)
$$

and similarly for the anti-quark jet function. The one-loop result for $J_{\mathrm{EEC}}^{q}$ is given in appendix A. This allows us to write our final factorized expression as

$$
\frac{d \sigma}{d z}=\frac{1}{2} \int d^{2} \vec{k}_{\perp} \int \frac{d^{2} \vec{b}_{\perp}}{(2 \pi)^{2}} e^{-i \vec{b}_{\perp} \cdot \vec{k}_{\perp}} H(Q, \mu) J_{\mathrm{EEC}}^{q}\left(\vec{b}_{\perp}, \mu, \nu\right) J_{\mathrm{EEC}}^{\bar{q}}\left(\vec{b}_{\perp}, \mu, \nu\right) S_{\mathrm{EEC}}\left(\vec{b}_{\perp}, \mu, \nu\right) \delta\left(1-z-\frac{\vec{k}_{\perp}^{2}}{Q^{2}}\right) .
$$

This provides a fully factorized description of the EEC in the back-to-back region into hard, jet and soft functions, and is one of the main results of this paper. We verify that this produces the known logarithmic structure at NNLO in appendix B.

We find it interesting that this factorization theorem of eq. (3.25) is as close as possible to a direct crossing of the factorization theorem for $q_{T}$ for color singlet production, ${ }^{4}$ which

\footnotetext{
${ }^{4}$ It would also be interesting to study semi-inclusive DIS with measured transverse momenta of an identified outgoing hadron. In this case, while it has been argued that the partially crossed soft function should be used [93, 94], the analysis of [95] indicates that future pointing Wilson lines should be used. We leave a study of this question in our framework to future work. We thank John Collins for discussions on this point.
} 
can be written in impact parameter space as

$$
\frac{1}{\sigma} \frac{d^{3} \sigma}{d^{2} \vec{q}_{T} d Y d Q^{2}}=H(Q, \mu) \int \frac{d^{2} \vec{b}_{\perp}}{(2 \pi)^{2}} e^{i \vec{b}_{\perp} \cdot \vec{q}_{T}}[B \times B]\left(\vec{b}_{\perp}, \mu, \nu\right) S_{\perp}\left(\vec{b}_{\perp}, \mu, \nu\right) .
$$

Here, instead of TMDFFs, transverse momentum dependent beam functions (also known as TMDPDFs) appear, and the soft function, referred to as the TMD soft function, is identical to the EEC soft function up to the direction of the Wilson lines in its definition. Explicitly, for the soft function, we have

$$
S_{\perp}\left(\vec{b}_{\perp}, \mu, \nu\right)=\lim _{\nu \rightarrow+\infty} \frac{1}{N_{c}} \operatorname{tr}\left\langle 0\left|T\left[S_{\bar{n}-}^{\dagger}(0) S_{n+}(0)\right] \bar{T}\left[S_{n-}^{\dagger}\left(y_{\nu}\left(\vec{b}_{\perp}\right)\right) S_{\bar{n}+}\left(y_{\nu}\left(\vec{b}_{\perp}\right)\right)\right]\right| 0\right\rangle .
$$

The precise definitions of the beam functions will not be important for the present discussion, but can be found in [91, 96, 97].

The key reason for the utility of this factorization theorem of eq. (3.25) is that all the ingredients are related (or identical) to other functions that have been calculated to high perturbative accuracy, namely the TMDFFs, and the TMD soft function. This will allow us to directly use these results to improve the perturbative understanding of the EEC observable. This ability to relate different functions highlights a benefit of operator based factorization theorems.

\subsection{Renormalization group evolution}

Large logarithms in the back-to-back region can be resummed by the renormalization group evolution of the functions appearing in the factorization theorem of eq. (3.25). Since this factorization theorem is constructed from well known objects, namely TMDFFs and the TMD soft function, we can immediately write down their renormalization group evolution. The $q_{T}$ dependent beam function and soft function were computed in the $\eta$ regulator of $[23,25]$ to NNLO [98]. The NNLO TMDPDF and soft function were calculated in $[96,97,99]$. The unpolarized TMDFF at NNLO was calculated in [84], from which it is possible to obtain the EEC jet function using eq. (3.24). The $q_{T}$ dependent beam and jet functions will be calculated in the exponential regulator of [40] that was used for the calculation of the three-loop soft function for color singlet $q_{T}$ [34] in a future publication [100].

The hard function satisfies a multiplicative RGE in $\mu$

$$
\mu \frac{d}{d \mu} H(Q, \mu)=2\left[\Gamma^{\mathrm{cusp}}\left(\alpha_{s}\right) \ln \frac{Q^{2}}{\mu^{2}}+\gamma^{H}\left(\alpha_{s}\right)\right] H(Q, \mu),
$$

and is independent of $\nu$. Here $\Gamma_{\text {cusp }}$ is the cusp anomalous dimension [101] (which is known analytically to three-loop order [102], and numerically to four loops [103]), and $\gamma^{H}$ is the non-cusp anomalous dimension of hard function, which can be found, for example, in [96]. The hard function is independent of the IR measurement, and its anomalous dimension can be obtained from the quark form factor, which is known to three-loops [104-106]. Since the result is well known (see e.g. [27]), we do not explicitly give it here. 
The EEC soft function satisfies RG equations in $\mu$

$$
\mu \frac{d S_{\mathrm{EEC}}\left(\vec{b}_{\perp}, \mu, \nu\right)}{d \mu}=\left[2 \Gamma_{\mathrm{cusp}}\left(\alpha_{s}\right) \ln \frac{\mu^{2}}{\nu^{2}}-2 \gamma_{\mathrm{EEC}}^{s}\left(\alpha_{s}\right)\right] S_{\mathrm{EEC}}\left(\vec{b}_{\perp}, \mu, \nu\right)
$$

and in $\nu[23,25,34]$

$$
\nu \frac{d S_{\mathrm{EEC}}\left(\vec{b}_{\perp}, \mu, \nu\right)}{d \nu}=2\left[-\int_{b_{0}^{2} / \vec{b}_{\perp}^{2}}^{\mu^{2}} \frac{d \bar{\mu}^{2}}{\bar{\mu}^{2}} \Gamma_{\mathrm{cusp}}\left(\alpha_{s}(\bar{\mu})\right)+\gamma_{\mathrm{EEC}}^{r}\left(\alpha_{s}\left(b_{0} /\left|\vec{b}_{\perp}\right|\right)\right)\right] S_{\mathrm{EEC}}\left(\vec{b}_{\perp}, \mu, \nu\right) .
$$

The anomalous dimensions $\gamma_{\mathrm{EEC}}^{s}$ and $\gamma_{\mathrm{EEC}}^{r}$ are known perturbatively to three-loops, and will be given in section 4.1 .

The matching coefficients, $\mathcal{I}_{i k}$, for the TMDFFs satisfy the $\mu \mathrm{RG}$

$$
\begin{aligned}
\mu \frac{d \mathcal{I}_{i k}\left(\vec{b}_{\perp} / z, z, \mu, \nu\right)}{d \mu}= & {\left[-\Gamma_{\text {cusp }}\left(\alpha_{s}\right) \ln \frac{Q^{2}}{\nu^{2}}+2 \gamma_{\mathrm{EEC}}^{J}\left(\alpha_{s}\right)\right] \mathcal{I}_{i k}\left(\vec{b}_{\perp} / z, z, \mu, \nu\right) } \\
& -2 \sum_{j} \mathcal{I}_{i j}\left(\vec{b}_{\perp} / z, z, \mu, \nu\right) \otimes P_{j k}\left(z, \alpha_{s}\right)
\end{aligned}
$$

where the convolution is defined as

$$
A(x) \otimes B(x)=\int_{0}^{1} d y \int_{0}^{1} d z \delta(x-y z) A(y) B(z) .
$$

Note that the coefficient functions depend on the impact parameter through $\vec{b}_{\perp} / z$ in the argument. The additional $1 / z$ factor compared with the more traditional TMDFF evolution comes from different convention in the normalization of fragmentation function. ${ }^{5}$ Additional discussion on this point will be presented in ref. [100]. The $\nu$ RG is given by

$$
\nu \frac{d \mathcal{I}_{i k}\left(\vec{b}_{\perp} / z, z, \mu, \nu\right)}{d \nu}=\left[\int_{b_{0}^{2} / \vec{b}_{\perp}^{2}}^{\mu^{2}} \frac{d \bar{\mu}^{2}}{\bar{\mu}^{2}} \Gamma_{\mathrm{cusp}}\left(\alpha_{s}(\bar{\mu})\right)-\gamma_{\mathrm{EEC}}^{r}\left(\alpha_{s}\left(b_{0} /\left|\vec{b}_{\perp}\right|\right)\right)\right] \mathcal{I}_{i k}\left(\vec{b}_{\perp} / z, z, \mu, \nu\right) .
$$

Here $P_{j k}$ are the time-like $j \rightarrow k$ splitting functions, which are known to three loops $[102,107]$ and for the non-singlet case to four loops in the large $N_{c}$ limit [103]. The anomalous dimension $\gamma_{\text {EEC }}^{J}$ is also known to three-loops due to the consistency of the factorization, as will be discussed shortly.

Using the known RG evolution equations for the TMDFFs we can derive the RG evolution equations for the jet function $J_{\mathrm{EEC}}^{q}\left(\vec{b}_{\perp}, \mu, \nu\right)$ appearing in our factorization formula

\footnotetext{
${ }^{5}$ We thank Alexey Vladimirov for pointing out to us the standard though unusual normalization of the TMD fragmentation functions.
} 
for the EEC. We have

$$
\begin{aligned}
\mu \frac{d J_{\mathrm{EEC}}^{q}\left(\vec{b}_{\perp}, \mu, \nu\right)}{d \mu}=\sum_{k} \int_{0}^{1} d x x\{[ & \left.-\Gamma_{\text {cusp }}\left(\alpha_{s}\right) \ln \frac{Q^{2}}{\nu^{2}}+2 \gamma_{\mathrm{EEC}}^{J}\left(\alpha_{s}\right)\right] \mathcal{I}_{q k}\left(\vec{b}_{\perp} / x, x, \mu, \nu\right) \\
& \left.-2 \sum_{j} \mathcal{I}_{q j}\left(\vec{b}_{\perp} / x, x, \mu, \nu\right) \otimes P_{j k}\left(x, \alpha_{s}\right)\right\} .
\end{aligned}
$$

The second line of eq. (3.34) can be simplified to

$$
\begin{aligned}
& -2 \sum_{k} \int_{0}^{1} d x x \sum_{j} \int d y d z \mathcal{I}_{q j}\left(\vec{b}_{\perp} / y, y, \mu, \nu\right) P_{j k}\left(z, \alpha_{s}\right) \delta(x-y z) \\
& =-2 \sum_{j} \int d y y \mathcal{I}_{q j}\left(\vec{b}_{\perp} / y, y, \mu, \nu\right) \sum_{k} \int d z z P_{j k}\left(z, \alpha_{s}\right) \\
& \quad=0
\end{aligned}
$$

where in the last line we have applied the momentum conservation sum rule for the time-like splitting function,

$$
\sum_{j} \int_{0}^{1} d x x P_{i j}(x)=0 .
$$

The $\mu$ RG for the jet function now simplifies to

$$
\mu \frac{d J_{\mathrm{EEC}}^{q}\left(\vec{b}_{\perp}, \mu, \nu\right)}{d \mu}=\left[-\Gamma_{\text {cusp }}\left(\alpha_{s}\right) \ln \frac{Q^{2}}{\nu^{2}}+2 \gamma_{\mathrm{EEC}}^{J}\left(\alpha_{s}\right)\right] J_{\mathrm{EEC}}^{q}\left(\vec{b}_{\perp}, \mu, \nu\right) .
$$

The anomalous dimensions for the quark and anti-quark jet functions are identical, and therefore we will simply use the notation $\gamma_{\text {EEC }}^{J}$ for both.

The $\nu$ RG for $J_{\mathrm{EEC}}^{q}$ follows trivially from the $\nu$ RG for $\mathcal{I}_{i j}$ in eq. (3.33), since it does not involve evolution in the momentum fraction. We therefore have

$$
\nu \frac{d J_{\mathrm{EEC}}^{q}\left(\vec{b}_{\perp}, \mu, \nu\right)}{d \nu}=\left[\int_{b_{0}^{2} / \vec{b}_{\perp}^{2}}^{\mu^{2}} \frac{d \bar{\mu}^{2}}{\bar{\mu}^{2}} \Gamma_{\text {cusp }}\left(\alpha_{s}(\bar{\mu})\right)-\gamma_{\mathrm{EEC}}^{r}\left(\alpha_{s}\left(b_{0} /\left|\vec{b}_{\perp}\right|\right)\right)\right] J_{\mathrm{EEC}}^{q}\left(\vec{b}_{\perp}, \mu, \nu\right) .
$$

From the RG invariance of the total cross section, we can immediately derive several relations between the different anomalous dimensions. For the $\mu$ anomalous dimensions, we have

$$
\frac{1}{2} \gamma^{H}+\gamma_{\mathrm{EEC}}^{J}-\frac{1}{2} \gamma_{\mathrm{EEC}}^{s}=0
$$

We have already used the consistency relations for the $\nu$ anomalous dimension in writing eqs. (3.30) and (3.33), where the same $\gamma_{\mathrm{EEC}}^{r}$ appears in both functions. This implies that the hard anomalous dimension, which is known and observable independent, combined with the soft anomalous dimension, which will be given in section 4 , are sufficient to determine $\gamma_{\mathrm{EEC}}^{J}$, and hence to completely fix the renormalization group evolution for all functions required to describe the EEC in the back-to-back region. 


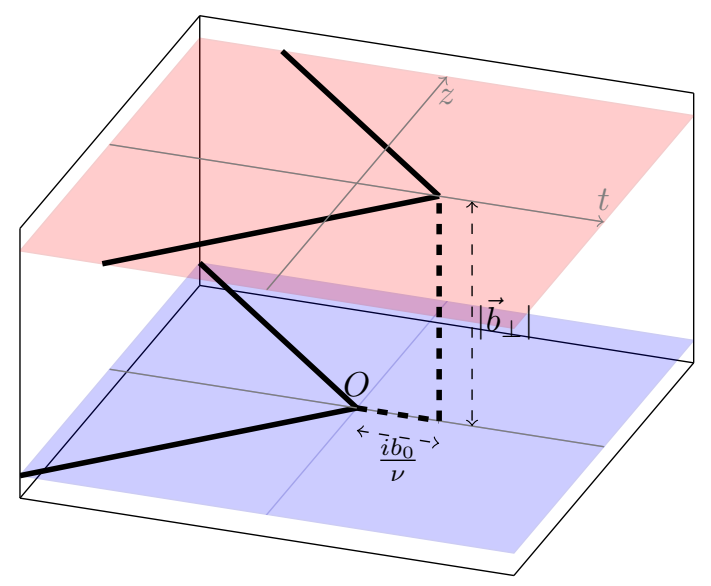

(a)

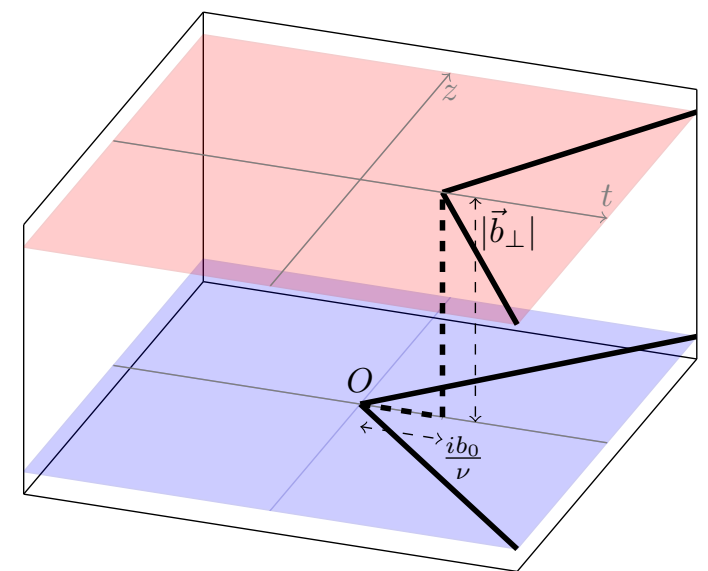

(b)

Figure 3. The soft functions for $q_{T}$ for color singlet production in (a) and for the EEC in (b), as defined in the exponential rapidity regulator of [40]. Solid black lines denotes Wilson lines. Both the rapidity regulator and the measurement are defined in terms of space-time shifts of the Wilson lines, allowing an all orders proof of the equivalence of these two soft functions.

\section{Three-loop anomalous dimensions and soft function}

As was noted earlier, the factorization theorem of eq. (3.25), which describes the singular structure of the EEC observable in the back-to-back limit, is closely related to the factorization theorem for $q_{T}$ for color singlet production given in eq. (3.26). In particular, the soft functions are identical up to the directions of the Wilson lines, as illustrated in figure 3. In this section, we study the relationship between the soft functions for the EEC and for $q_{T}$. In section 4.1, we use this relation to give the three-loop $\mu$ and $\nu$ anomalous dimensions for the EEC soft function, using the recently calculated results for the $q_{T}$ soft function. In section 4.2 we prove the equivalence of the soft function for $q_{T}$ and the EEC to all orders, i.e. the independence of the soft function on crossing the directions of the Wilson lines, which allows us to give the three-loop finite terms of the soft function for the EEC.

\subsection{Anomalous dimensions}

The anomalous dimensions for the soft function are independent of the direction of the Wilson lines. This can be proven following [92], by noting that the jet and beam function (TMDFF and TMDPDF) anomalous dimensions can be proven to be equal from their operator definitions. The consistency relations for the anomalous dimensions in eq. (3.39) then imply that the anomalous dimensions of the soft function must be independent of the directions of the Wilson lines to all orders. We therefore have the relations

$$
\gamma_{\mathrm{EEC}}^{r}=\gamma_{q_{T}}^{r} \equiv \gamma^{r}, \quad \gamma_{\mathrm{EEC}}^{s}=\gamma_{q_{T}}^{s} \equiv \gamma^{s} .
$$

Due to their equivalence, and to simplify our notation, we will drop the subscripts and simply write $\gamma^{r}$ and $\gamma^{s}$. The one- and two-loop anomalous dimensions were calculated long 
ago [108-110], while the three-loop anomalous dimensions were calculated quite recently. We expand the anomalous dimensions perturbatively as

$$
\gamma^{r}=\sum_{n \geq 0}\left(\frac{\alpha_{s}}{4 \pi}\right)^{n+1} \gamma_{n}^{r}, \quad \gamma^{s}=\sum_{n \geq 0}\left(\frac{\alpha_{s}}{4 \pi}\right)^{n+1} \gamma_{n}^{s} .
$$

The soft anomalous dimensions up to three-loops are [111]

$$
\begin{aligned}
\gamma_{0}^{s}= & 0, \\
\gamma_{1}^{s}= & C_{A} C_{F}\left(-\frac{808}{27}+\frac{22}{3} \zeta_{2}+28 \zeta_{3}\right)+C_{F} n_{f}\left(\frac{112}{27}-\frac{4}{3} \zeta_{2}\right), \\
\gamma_{2}^{s}= & C_{A}^{2} C_{F}\left(-\frac{136781}{729}+\frac{12650}{81} \zeta_{2}+\frac{1316}{3} \zeta_{3}-176 \zeta_{4}-192 \zeta_{5}-\frac{176}{3} \zeta_{3} \zeta_{2}\right) \\
& +C_{A} C_{F} n_{f}\left(\frac{11842}{729}-\frac{2828}{81} \zeta_{2}-\frac{728}{27} \zeta_{3}+48 \zeta_{4}\right) \\
& +C_{F}^{2} n_{f}\left(\frac{1711}{27}-4 \zeta_{2}-\frac{304}{9} \zeta_{3}-16 \zeta_{4}\right)+C_{F} n_{f}^{2}\left(\frac{2080}{729}+\frac{40}{27} \zeta_{2}-\frac{112}{27} \zeta_{3}\right) .
\end{aligned}
$$

The rapidity anomalous dimensions up to three-loops are [34]

$$
\begin{aligned}
\gamma_{0}^{r}= & 0 \\
\gamma_{1}^{r}= & C_{F} C_{A}\left(-\frac{808}{27}+28 \zeta_{3}\right)+C_{F} n_{f} \frac{112}{27}, \\
\gamma_{2}^{r}= & C_{F} C_{A}^{2}\left(-\frac{297029}{729}+\frac{6392}{81} \zeta_{2}+\frac{12328}{27} \zeta_{3}+\frac{154}{3} \zeta_{4}-192 \zeta_{5}-\frac{176}{3} \zeta_{3} \zeta_{2}\right) \\
& +C_{F} C_{A} n_{f}\left(\frac{62626}{729}-\frac{824}{81} \zeta_{2}-\frac{904}{27} \zeta_{3}+\frac{20}{3} \zeta_{4}\right)+C_{F} n_{f}^{2}\left(-\frac{1856}{729}-\frac{32}{9} \zeta_{3}\right) \\
& +C_{F}^{2} n_{f}\left(\frac{1711}{27}-\frac{304}{9} \zeta_{3}-16 \zeta_{4}\right) .
\end{aligned}
$$

Following the original calculation of the three-loop result in [34], this result was verified in [49] using an equivalence between rapidity and virtuality anomalous dimensions [49, 50]. Using the consistency relations of eq. (3.39), along with the known result for the hard anomalous dimension, this completely determines all anomalous dimensions governing the RG evolution of the EEC in the back-to-back region, and allows for resummation to $\mathrm{N}^{3} \mathrm{LL}$.

Interestingly, in planar $\mathcal{N}=4 \mathrm{SYM}$ the result for the rapidity anomalous dimension can be extended to higher orders. Using the equivalence between the rapidity anomalous dimension and the eikonal collinear anomalous dimension [49, 50], we can use the results of [112] to relate $\gamma^{r}$ and the collinear anomalous dimension $\mathcal{G}_{0}$ as

$$
\gamma^{r}=-\mathcal{G}_{0}+2 B
$$

Here $B$ is the virtual anomalous dimension, i.e. the coefficient of $\delta(1-x)$ in the DGLAP kernel. It is known to all orders in planar $\mathcal{N}=4 \mathrm{SYM}$ using integrability [113-115]. Remarkably, the collinear anomalous dimension was recently analytically computed to 
four-loops [116] (it was computed numerically to four-loops in [117]), providing also the rapidity anomalous dimension for the EEC at this order. The knowledge of these anomalous dimensions to such high orders, along with the hope that they may be computed to all orders using integrability, makes the EEC an interesting playground for studying the perturbative structure of resummation at high orders for a physical observable.

\subsection{Equivalence of soft functions and the three-loop boundary condition}

While the anomalous dimensions of the soft functions are independent of the direction of the Wilson lines, this is not in general true for the full soft function due to the presence of Glauber modes. At one- and two-loops, it has been shown that a wide class of soft functions are independent of the directions of the Wilson lines [118]. This result can also be seen using an EFT approach [119] that allows the Glauber region to be separated. Up to two-loops, Glaubers contribute at most a phase $(i \pi)$, which cancels out of the squared amplitude. However, starting at three-loops, which is the order of interest in the current paper, such Glauber contributions could begin to contribute, making the soft function depend on the direction of the Wilson lines. For a general soft function, this must be assumed to be true. To be able to achieve $\mathrm{N}^{3} \mathrm{LL}$ ' accuracy (where the prime denotes the inclusion of the three-loop boundary condition for the soft and collinear functions, see e.g. [73] for a discussion of order counting), we would like to understand whether or not the $q_{T}$ and EEC soft functions are identical. ${ }^{6}$

It was argued in [120-122] that the $q_{T}$ soft function is independent of the direction of the Wilson lines. ${ }^{7}$ While we agree with the conclusions of [120-122], details related to the time ordering and the regularization of the matrix element, which can lead to subtleties, were not made explicit. ${ }^{8}$ Here we will use the exponential regulator of $[34,40]$ to prove the independence of the soft function on crossing the Wilson line directions. However, we still believe that it would be an interesting exercise to explicitly compute the Glauber contributions in the EFT approach of [119] to understand their invariance under the crossing of the Wilson lines. Some of the required integrals were performed in [130].

Our proof of the all orders equivalence of the soft function is specific to the $q_{T}$ soft function with Wilson lines along back-to-back directions, as well as to the particular form of the regulator of $[34,40]$. Most importantly, both the measurement function, and the regulator take the form of a spacetime shift on the Wilson lines appearing in the soft

\footnotetext{
${ }^{6}$ We note that the fact that Glaubers do not contribute to the EEC or color singlet $q_{T}$ distributions is a distinct statement from whether or not the soft function is independent of the Wilson line directions. The statement that Glaubers cancel in a physical observable should be more precisely stated as the fact that they can be absorbed into the soft or collinear sectors by an appropriate choice of Wilson line directions. In the language of CSS, this is the statement of whether contours can be deformed out of the Glauber region, and in the EFT language it is related to whether the choice of Wilson line direction can be made such that their is a cancellation between the Glauber zero-bin and the Glaubers themselves. Since these proofs force a direction of the Wilson lines, they cannot also be used to prove independence on the direction of the Wilson lines. This would amount to circular reasoning.

${ }^{7}$ Arguments similar to those presented here, using spacetime symmetries to relate soft and collinear functions have been discussed in great detail in [120-128], particularly in relation to the Sivers effect [129].

${ }^{8}$ For more detailed discussions and an alternative treatment of these issues to that presented here, see [83].
} 
function. This is shown in figure 3. This is specific to the $q_{T}$ measurement function, and also allows the regulator to be formulated to all orders (and non-perturbatively) greatly simplifying the proof. $^{9}$

For convenience, we recall the definitions of the soft functions for $q_{T}$ and the EEC. For the case of the EEC, we have

$$
S_{\mathrm{EEC}}\left(\vec{b}_{\perp}, \mu, \nu\right)=\lim _{\nu \rightarrow+\infty} \frac{\operatorname{tr}}{N_{c}}\left\langle 0\left|T\left[S_{\bar{n}+}^{\dagger}(0) S_{n-}(0)\right] \bar{T}\left[S_{n+}^{\dagger}\left(y_{\nu}\left(\vec{b}_{\perp}\right)\right) S_{\bar{n}-}\left(y_{\nu}\left(\vec{b}_{\perp}\right)\right)\right]\right| 0\right\rangle,
$$

and for the case of the color-singlet $q_{T}$ soft function

$$
S_{\perp}\left(\vec{b}_{\perp}, \mu, \nu\right)=\lim _{\nu \rightarrow+\infty} \frac{\operatorname{tr}}{N_{c}}\left\langle 0\left|T\left[S_{\bar{n}-}^{\dagger}(0) S_{n+}(0)\right] \bar{T}\left[S_{n-}^{\dagger}\left(y_{\nu}\left(\vec{b}_{\perp}\right)\right) S_{\bar{n}+}\left(y_{\nu}\left(\vec{b}_{\perp}\right)\right)\right]\right| 0\right\rangle .
$$

Again, we emphasize that due to the particular nature of the measurement, and the implementation of the regulator as a spacetime shift, this is a vacuum matrix element of (shifted) Wilson lines. Here we have also made the (anti-) time ordering explicit (For a discussion of the importance of the time-ordering, see [91]). The time ordering must be treated carefully, since when using time reversal arguments to flip the directions of the Wilson lines, the time ordering also flips, as can be seen for a simple bosonic field

$$
\begin{aligned}
T\left[\phi\left(t_{1}\right) \phi\left(t_{2}\right)\right] & =\phi\left(t_{1}\right) \phi\left(t_{2}\right) \Theta\left(t_{1}-t_{2}\right)+\phi\left(t_{2}\right) \phi\left(t_{1}\right) \Theta\left(t_{2}-t_{1}\right) \\
& \stackrel{\mathrm{T}}{\rightarrow} \phi\left(-t_{1}\right) \phi\left(-t_{2}\right) \Theta\left(-t_{1}+t_{2}\right)+\phi\left(-t_{2}\right) \phi\left(-t_{1}\right) \Theta\left(-t_{2}+t_{1}\right) \\
& =\bar{T}\left[\phi\left(-t_{1}\right), \phi\left(-t_{2}\right)\right] .
\end{aligned}
$$

For general soft functions, where the regulator and measurement cannot be formulated as a shift, one has a time ordered matrix element squared, and the time ordering can disrupt the proof of Wilson line direction independence, as noted in [118]. However, as seen in eqs. (4.6) and (4.7), for our particular soft function of interest both time ordered and anti-time ordered contributions appear in the matrix element, which will exchange under time reversal.

To prove the universality of the soft function we start with the EEC soft function, and apply time reversal symmetry, ${ }^{10}$ using the fact that the vacuum states are invariant

$$
\begin{aligned}
S_{\mathrm{EEC}}\left(\vec{b}_{\perp}, \mu, \nu\right) & =\lim _{\nu \rightarrow+\infty} \frac{\operatorname{tr}}{N_{c}}\left\langle 0\left|T\left[S_{\bar{n}+}^{\dagger}(0) S_{n-}(0)\right] \bar{T}\left[S_{n+}^{\dagger}\left(y_{\nu}\left(\vec{b}_{\perp}\right)\right) S_{\bar{n}-}\left(y_{\nu}\left(\vec{b}_{\perp}\right)\right)\right]\right| 0\right\rangle \\
& \stackrel{\mathrm{T}}{\rightarrow} \lim _{\nu \rightarrow+\infty} \frac{\operatorname{tr}}{N_{c}}\left\langle 0\left|T\left[S_{n+}\left(y_{\nu}^{T}\left(\vec{b}_{\perp}\right)\right) S_{\bar{n}-}^{\dagger}\left(y_{\nu}^{T}\left(\vec{b}_{\perp}\right)\right)\right] \bar{T}\left[S_{\bar{n}+}(0) S_{n-}^{\dagger}(0)\right]\right| 0\right\rangle .
\end{aligned}
$$

Here the time reversal changes the displacement of the Wilson lines ${ }^{11}$

$$
\begin{aligned}
y_{\nu}\left(\vec{b}_{\perp}\right) & =\left(i b_{0} / \nu, i b_{0} / \nu, \vec{b}_{\perp}\right) \\
& \stackrel{\mathrm{T}}{\rightarrow} y_{\nu}^{T}\left(\vec{b}_{\perp}\right) \equiv\left(-i b_{0} / \nu,-i b_{0} / \nu, \vec{b}_{\perp}\right) .
\end{aligned}
$$

\footnotetext{
${ }^{9}$ In particular, it is much simpler than the case of hemisphere soft functions considered in [118], where the measurement function cannot in general be formulated as a spacetime shift.

${ }^{10}$ For a detailed discussion of the transformation properties of Wilson lines, see e.g. [83].

${ }^{11}$ We have used the superscript notation for the transformed vector to distinguish it from the time ordering operator.
} 
We can now use the translation invariance of the matrix element, combined with the fact that the soft function depends only on $\vec{b}_{\perp}^{2}$, to translate the arguments back to the original positions defining the $S_{\perp}$ soft function. This set of transformations can also easily be understood by looking at the positions of the two Wilson lines in figure 3. We therefore obtain

$$
S_{\mathrm{EEC}}\left(\vec{b}_{\perp}, \mu, \nu\right)=S_{\perp}\left(\vec{b}_{\perp}, \mu, \nu\right) .
$$

In summary the proof used that the measurement and regulator were formulated as spacetime shifts, that there were only two Wilson line directions, that the soft function is independent of $n \leftrightarrow \bar{n}$, depends only on $x=-\vec{b}_{\perp}^{2} \nu^{2} / b_{0}^{2}$, and that one has time reversal invariance, and translation invariance. It is therefore quite specific to the particular case of interest, and we do not make claims for more general soft functions.

Using this equivalence, and the recently computed three-loop result for the $q_{T}$ soft function [34], we can give the three-loop constant for the EEC soft function, which acts as the boundary condition for the RG evolution. Using the non-Abelian exponentiation theorem [131-133], which is preserved by the exponential regulator, we can write the soft function as

$$
S_{\mathrm{EEC}}\left(\vec{b}_{\perp}, \mu, \nu\right)=\exp \left[\left(\frac{\alpha_{s}}{4 \pi}\right) S_{1}^{\mathrm{EEC}}+\left(\frac{\alpha_{s}}{4 \pi}\right)^{2} S_{2}^{\mathrm{EEC}}+\left(\frac{\alpha_{s}}{4 \pi}\right)^{3} S_{3}^{\mathrm{EEC}}+\mathcal{O}\left(\alpha_{s}^{4}\right)\right] .
$$

The boundary conditions are then given by the soft function evaluated at its natural scales

$$
c_{i}^{\mathrm{EEC}} \equiv S_{i}^{\mathrm{EEC}}\left(\vec{b}_{\perp}, \mu=\frac{b_{0}}{\left|\vec{b}_{\perp}\right|}, \nu=\frac{b_{0}}{\left|\vec{b}_{\perp}\right|}\right) .
$$

Using the results of [34], we can now give the explicit result for the EEC soft function constant to three-loops:

$$
\begin{aligned}
c_{1}^{\mathrm{EEC}}= & -2 C_{F} \zeta_{2}, \\
c_{2}^{\mathrm{EEC}}= & C_{A} C_{F}\left(\frac{2428}{81}-\frac{67}{3} \zeta_{2}-\frac{154}{9} \zeta_{3}+10 \zeta_{4}\right) \\
& +C_{F} n_{f}\left(-\frac{328}{81}+\frac{10}{3} \zeta_{2}+\frac{28}{9} \zeta_{3}\right), \\
c_{3}^{\mathrm{EEC}}= & C_{F} C_{A}^{2}\left(\frac{5211949}{13122}-\frac{297481}{729} \zeta_{2}-\frac{151132}{243} \zeta_{3}+\frac{3649}{27} \zeta_{4}\right. \\
& \left.+\frac{1804}{9} \zeta_{5}+\frac{1100}{9} \zeta_{2} \zeta_{3}-\frac{3086}{27} \zeta_{6}+\frac{928}{9} \zeta_{3}^{2}\right) \\
& +C_{F} C_{A} n_{f}\left(-\frac{412765}{6561}+\frac{74530}{729} \zeta_{2}+\frac{8152}{81} \zeta_{3}-\frac{416}{27} \zeta_{4}-\frac{184}{3} \zeta_{5}+\frac{40}{9} \zeta_{3} \zeta_{2}\right) \\
& +C_{F}^{2} n_{f}\left(-\frac{42727}{486}+\frac{275}{9} \zeta_{2}+\frac{3488}{81} \zeta_{3}+\frac{152}{9} \zeta_{4}+\frac{224}{9} \zeta_{5}-\frac{80}{3} \zeta_{3} \zeta_{2}\right) \\
& +C_{F} n_{f}^{2}\left(-\frac{256}{6561}-\frac{136}{27} \zeta_{2}-\frac{560}{243} \zeta_{3}-\frac{44}{27} \zeta_{4}\right) .
\end{aligned}
$$


The full result for the soft function evaluated at a general scale is given in appendix B. This is an important ingredient for resummation to $\mathrm{N}^{3} \mathrm{LL}$ ' accuracy for the EEC. Here the superscript ' denotes the inclusion of the constant terms in the functions in the EFT. It is often found that this improves the matching to the NNLO fixed order result, see e.g. [27, 28]. This represents the state of the art for any event shape observable in QCD, and furthermore, this is the first time that this accuracy has been achieved for a recoil sensitive $\left(\mathrm{SCET}_{\mathrm{II}}\right) e^{+} e^{-}$event shape.

\section{Resummation formula}

The factorization theorem in eq. (3.25) can be used to resum all large logarithms of $1-z$ appearing in the back-to-back region through $\mathrm{RG}$ evolution in both rapidity and virtuality. By rotational symmetry, we can integrate out $\vec{k}_{\perp}$ and the angular component of $\vec{b}_{\perp}$, giving

$$
\frac{d \sigma}{d z}=\frac{1}{2} \int_{0}^{\infty} \frac{b d b}{2} J_{0}(b Q \sqrt{1-z}) H(Q, \mu) j_{\mathrm{EEC}}^{q}(b, \mu, \nu) j_{\mathrm{EEC}}^{\bar{q}}(b, \mu, \nu) S_{\mathrm{EEC}}(b, \mu, \nu)
$$

where $J_{0}(x)$ is the Bessel function of the first kind, and we have made it clear that the jet and soft functions only depend on the magnitude of $\vec{b}_{\perp}, b=\sqrt{\vec{b}_{\perp}^{2}}$.

Resummation can be achieved by setting the renormalization and rapidity separately for each of the factorized ingredient to minimize the large logarithms, and then evolving all scales to a common value. The natural scales for the hard, jet and soft functions are

$$
\mu_{h}=Q, \quad \mu_{j}=b_{0} / b, \quad \mu_{s}=b_{0} / b, \quad \nu_{j}=Q, \quad \nu_{s}=b_{0} / b
$$

Below we choose to evolve the hard function and soft function to the jet function scales. Other choices could also be used, as guaranteed by the consistency of the anomalous dimensions. The evolution for the hard function is

$$
H(Q, \mu)=H\left(Q, \mu_{h}\right) \exp \left[\int_{\mu_{h}^{2}}^{\mu^{2}} \frac{d \bar{\mu}^{2}}{\bar{\mu}^{2}}\left(\Gamma^{\mathrm{cusp}}\left(\alpha_{s}(\bar{\mu})\right) \ln \frac{Q^{2}}{\bar{\mu}^{2}}+\gamma_{H}\left(\alpha_{s}(\bar{\mu})\right)\right] .\right.
$$

For the soft function, we have evolution both in renormalization scale and rapidity scale,

$$
\begin{aligned}
S_{\mathrm{EEC}}(b, \mu, \nu)= & S_{\mathrm{EEC}}\left(b, \mu_{s}, \nu_{s}\right) \exp \left[\int_{\mu_{s}^{2}}^{\mu^{2}} \frac{d \bar{\mu}^{2}}{\bar{\mu}^{2}}\left(\Gamma^{\mathrm{cusp}}\left(\alpha_{s}(\bar{\mu})\right) \ln \frac{b^{2} \bar{\mu}^{2}}{b_{0}^{2}}-\gamma_{\mathrm{EEC}}^{s}\left(\alpha_{s}(\bar{\mu})\right)\right)\right. \\
& \left.+\ln \frac{\nu^{2}}{\nu_{s}^{2}}\left(-\int_{b_{0}^{2} / b^{2}}^{\mu^{2}} \frac{d \bar{\mu}^{2}}{\bar{\mu}^{2}} \Gamma^{\mathrm{cusp}}\left(\alpha_{s}(\bar{\mu})\right)+\gamma_{\mathrm{EEC}}^{r}\left(\alpha\left(b_{0} / b\right)\right)\right)\right] .
\end{aligned}
$$


Substituting eqs. (5.3) and (5.4) into eq. (5.1), and setting $\mu=b_{0} / b, \nu=Q$, we obtain

$$
\begin{aligned}
\frac{d \sigma}{d z}= & \frac{1}{4} \int_{0}^{\infty} d b b J_{0}(b Q \sqrt{1-z}) H\left(Q, \mu_{h}\right) j_{\mathrm{EEC}}^{q}\left(b, b_{0} / b, Q\right) j_{\mathrm{EEC}}^{\bar{q}}\left(b, b_{0} / b, Q\right) S_{\mathrm{EEC}}\left(b, \mu_{s}, \nu_{s}\right) \\
& \cdot\left(\frac{Q^{2}}{\nu_{s}^{2}}\right)^{\gamma_{\mathrm{EEC}}^{r}\left(\alpha_{s}\left(b_{0} / b\right)\right)} \exp \left[\int_{\mu_{s}^{2}}^{\mu_{h}^{2}} \frac{d \bar{\mu}^{2}}{\bar{\mu}^{2}} \Gamma^{\mathrm{cusp}}\left(\alpha_{s}(\bar{\mu})\right) \ln \frac{b^{2} \bar{\mu}^{2}}{b_{0}^{2}}\right. \\
& \left.+\int_{\mu_{h}^{2}}^{b_{0}^{2} / b^{2}} \frac{d \bar{\mu}^{2}}{\bar{\mu}^{2}}\left(\Gamma^{\mathrm{cusp}}\left(\alpha_{s}(\bar{\mu})\right) \ln \frac{b^{2} Q^{2}}{b_{0}^{2}}+\gamma^{H}\left(\alpha_{s}(\bar{\mu})\right)\right)-\int_{\mu_{s}^{2}}^{b_{0}^{2} / b^{2}} \frac{d \bar{\mu}^{2}}{\bar{\mu}^{2}} \gamma_{\mathrm{EEC}}^{s}\left(\alpha_{s}(\bar{\mu})\right)\right]
\end{aligned}
$$

Eq. (5.5) gives our final formula for the resummation of large logarithms of $1-z$ for the EEC in the back-to-back region, and is another main result of this paper. It shows the all orders resummation of logarithms of $1-z$, and we have given field theoretic definitions for all ingredients appearing in the formula, in particular, for the anomalous dimensions $\gamma_{\mathrm{EEC}}^{r}$, $\gamma_{\text {EEC }}^{S}$, and $\gamma^{H}$, which control the renormalization group evolution. At each perturbative order, remaining scale uncertainties are estimated by varying $\mu_{h}, \mu_{s}$, and $\nu_{s}$ around their nominal values.

Here we have performed the resummation directly in impact parameter space. There has been recent work on understanding the resummation of $q_{T}$ sensitive observables in momentum space [134-136]. This has been done in [134] using a coherent branching type formalism [17, 18], and in [135] by solving distributional evolution equations. We hope that the particularly simple form of the resummation for the EEC, and the fact that it is a non-perturbatively well defined observable even in a conformal theory, may allow it to be a useful observable for studying many of these issues.

Finally, we note that we have considered in this section only the perturbative distribution. Non-perturbative corrections to the EEC have been studied in [10, 16, 62, 66]. An important aspect of our factorization theorem is the operator definitions of the jet and soft functions that describe the dynamics of the EEC in the back-to-back limit. This enables non-perturbative effects to be studied, and related to other observables, in particular, $q_{T}$. Conversely, there has been significant interest in the non-perturbative functions appearing in the description of the $q_{T}$ distribution, such as $g_{K}\left(b_{T}\right)$ (see e.g. [137] for definitions and a recent discussion), which is closely related to our rapidity anomalous dimenion $\gamma_{\mathrm{EEC}}^{r}$. The fact that these functions also appear in the EEC, which is an inclusive event shape, may facilitate their study.

\section{Conclusions}

In this paper we have presented an analytic result for the three-loop soft function for the EEC observable in the back-to-back region. This result was derived from a new factorization theorem describing the leading power dynamics in the back-to-back region, whose soft function is identical to the case of $q_{T}$ for color singlet production up to the direction of the Wilson lines. This factorization theorem provides an operator level correspondence between the EEC observable, and $q_{T}$, which is the most important advantage of our approach compared to approaches taken previously in the literature, for example in ref. [10]. In ref. [10], 
the NNLL resummation formula is established by matching a CSS like formula with the single logarithmic term at $\mathcal{O}\left(\alpha_{s}^{2}\right)$ from an explicit two-loop perturbative calculation. In our formula, predictions at NNLL accuracy are fully determined using one-loop matching calculation for the soft and jet function, and the well-known anomalous dimensions from $q_{T}$ resummation, thanks to the correspondence between Drell-Yan and $e^{+} e^{-}$process as was explained in section 4. Furthermore, our formula can also predict the coefficient of $\delta(1-z)$. An explicit example at NLO is given in the appendix B. Our factorization theorem thus enables the resummation of all large logarithms appearing in the perturbative expansion of the EEC in the back-to-back region beyond NNLL, and we provided analytic results for all anomalous dimensions to three-loop order, allowing resummation to $\mathrm{N}^{3} \mathrm{LL}$.

The EEC is now the $q_{T}$ sensitive $\left(\mathrm{SCET}_{\mathrm{II}}\right)$ observable about which the highest order perturbative information is known, making it a prime candidate for precision extractions of $\alpha_{s}$ from LEP data, which will complement those from $\mathrm{SCET}_{\mathrm{I}}$ observables. This has already been pursued recently in ref. [16] at NNLL matched to NNLO, and it would be interesting to improve the perturbative precision to $\mathrm{N}^{3} \mathrm{LL}$. In addition to the anomalous dimensions presented here, the full calculation at $\mathrm{N}^{3} \mathrm{LL}+\mathrm{NNLO}$ will also require the calculation of the NNLO jet functions. This can be accomplished by crossing ingredients used in the calculation of the transverse momentum dependent beam functions, and results with the exponential regulator used here will be presented in a future publication [100]. Along similar lines, the distinct perturbative and non-perturbative structure as compared with recoil free observables will make the comparison of precision calculations for the EEC with Monte Carlo parton shower programs useful for improving the modeling of quark and gluon jets, as was considered for thrust in [15].

The exceptional perturbative control of both the EEC and color singlet $q_{T}$ spectrum motivates an improved understanding of non-perturbative effects for $q_{T}$ sensitive observables. While non-perturbative effects have been studied for broadening [138], $q_{T}[108,109,138-140]$, groomed fragmentation [141], semi-inclusive DIS [142], and the EEC $[10,16,62,66]$, it has been found in a variety of studies that the standard shape function parametrizations used were not sufficient to describe non-perturbative effects $[10,16]$. It will be essential to achieve an improved understanding for precision extractions of $\alpha_{s}$, and we hope that this will also help in understanding the non-perturbative corrections for the $q_{T}$ spectrum.

There are a number of additional directions that will be interesting to pursue involving the EEC. In the $\chi \rightarrow 0$ limit, the EEC can be calculated at LL accuracy using the jet calculus [143] (see also [69]), however, it would also be interesting to formulate an operator based factorization theorem in terms of jet and soft functions, to allow improved perturbative control in this limit. It may also be interesting to study higher point energy-energy correlations in $e^{+} e^{-}$. This has been done successfully in jet substructure [144-149], but could hopefully be done in a manner which preserves the simple perturbative structure of the EEC. Finally, the simplicity of the EEC observable may also prove useful for the study of the analytic structure of fixed order corrections to perturbative event shapes, and of their perturbative power corrections [150-155]. We hope that these many interesting directions can generate renewed interest in the EEC observable. 


\section{Acknowledgments}

We thank Lance Dixon for bringing the EEC observable to our attention, as well as for discussions regarding his $\mathcal{N}=4$ results for the collinear anomalous dimension, and Duff Neill for helpful discussions and references regarding the universality of the soft function and the role of Glaubers. We thank Tong-Zhi Yang for checking some of the equations in this paper. We thank Alexey Vladimirov, John Collins, Lance Dixon, Anjie Gao and Markus Ebert for helpful comments on the first draft, as well as for catching a number of typos. I.M. thanks Zhejiang University for hospitality while portions of this work were performed. I.M. was supported in part by the Office of High Energy Physics of the U.S. Department of Energy under Contract No. DE-AC02-05CH11231, and the LDRD Program of LBNL. H.X.Z. was supported in part by the Thousand Youth Program of China under contract 588020-X01702/076.

\section{A Matching coefficients for the TMDFF and EEC jet function}

The matching coefficient $\mathcal{I}_{j i}\left(x, \vec{k}_{\perp}\right)$ from the TMDFF to the conventional fragmentation function can be calculated perturbatively as the probability of finding a parton $i$ from a parton $j$, with momentum fraction $x$ and transverse momentum $\vec{k}_{\perp}$ relative to the partonic jet axis, which is aligned with the total jet three momentum. At LO the matching coefficients are trivial,

$$
\mathcal{I}_{j i}\left(x, \vec{b}_{\perp}\right)= \begin{cases}\delta(1-x) & \text { if } i=j, \\ 0 & \text { if } i \neq j .\end{cases}
$$

At NLO, the matching coefficients before zero-bin subtraction [156] can be calculated from the LO splitting kernel $P_{\widetilde{i k}} \rightarrow p_{i} k$, where $p_{i}$ and $k$ are on-shell momentum. Explicitly, we have

$$
\begin{aligned}
\frac{\alpha_{s}}{4 \pi} \mathcal{I}_{j i}^{(1), \text { bare }}= & \frac{1}{z} \mu^{2 \epsilon} \lim _{\tau \rightarrow 0} \int \frac{\mathrm{d}^{4-2 \epsilon} k}{(2 \pi)^{3-2 \epsilon}} \Theta\left(k^{0}\right) \delta\left(k^{2}\right) \delta\left(\frac{k^{-}}{Q}-(1-z)\right) g_{s}^{2} \frac{1}{s_{i k}} p_{j i}^{(0)}(z, \epsilon) \\
& \cdot \exp \left[-\frac{b_{0} \tau}{2}\left(k^{+}+k^{-}\right)+i \vec{b}_{\perp} \cdot \vec{k}_{\perp}\right]
\end{aligned}
$$

where $\tau=1 / \nu, b_{0}=2 e^{-\gamma_{E}}$, and $Q=P_{\widetilde{i k}}^{-}$is the label momentum of the jet. The $1 / z$ factor comes from phase space factorization, and $s_{i k}=\left(p_{i}+k\right)^{2}=\vec{k}_{\perp}^{2} /(z(1-z))$. For the quark $\mathrm{FF}$, the relevant splitting kernel are

$$
\begin{aligned}
& p_{q q}^{(0)}(z, \epsilon)=2 C_{F}\left[\frac{1+z^{2}}{1-z}-\epsilon(1-z)\right], \\
& p_{q g}^{(0)}(z, \epsilon)=p_{q q}(1-z, \epsilon) .
\end{aligned}
$$

The integral in eq. (A.2) can be done analytically in the limit of $\tau \rightarrow 0$, keeping only the leading power terms in $\tau$. The results are

$$
\begin{aligned}
& \widetilde{\mathcal{I}}_{q q}^{(1)}=C_{F}\left(-2 L_{\perp}^{2}-2 L_{\perp} L_{Q}-4 L_{\perp} L_{\nu}+3 L_{\perp}-\frac{\pi^{2}}{3}\right) \delta(1-z)-C_{F} L_{\perp} P_{q q}^{(0)}(z)+2 C_{F}(1-z), \\
& \widetilde{\mathcal{I}}_{q g}^{(1)}=C_{F}\left(-L_{\perp} P_{q g}^{(0)}(z)+2 z\right)
\end{aligned}
$$


where

$$
\begin{aligned}
& P_{q q}^{(0)}(z)=3 \delta(1-z)+2 \frac{1+z^{2}}{[1-z]_{+}}, \\
& P_{q g}^{(0)}(z)=\frac{4-4 z+2 z^{2}}{z} .
\end{aligned}
$$

Note that there is no need to regularize $P_{q g}^{(0)}(z)$ in eq. (A.5), since in the jet function it is weighted by $z$ in the numerator. In eq. (A.4) we have defined

$$
L_{\perp}=\ln \frac{\vec{b}_{\perp}^{2} \mu^{2}}{b_{0}^{2}}, \quad L_{\nu}=\ln \frac{\nu^{2}}{\mu^{2}}, \quad L_{Q}=\ln \frac{Q^{2}}{\nu^{2}} .
$$

The results in eq. (A.4) have a non-trivial zero-bin. In the exponential regularization scheme [40], the zero-bin is the same as the soft function. The zero-bin can be straightforwardly removed by dividing the fragmentation function by the soft function. We find that the zero-bin subtracted TMDFF coefficients are

$$
\begin{aligned}
& \left.\mathcal{I}_{q q}^{(1)}=C_{F}\left(-2 L_{\perp} L_{Q}+3 L_{\perp}\right) \delta(1-z)-L_{\perp} P_{q q}^{(0)}(z)+2(1-z)\right), \\
& \mathcal{I}_{q g}^{(1)}=\widetilde{\mathcal{I}}_{q g}^{(1)}=C_{F}\left(-L_{\perp} P_{q g}^{(0)}(z)+2 z\right) .
\end{aligned}
$$

Using these results we can compute the tree level and one-loop result for the jet function appearing in the EEC factorization theorem. Recall that it was defined as

$$
J_{\mathrm{EEC}}^{q}\left(\vec{b}_{\perp}\right)=\sum_{i} \int_{0}^{1} d x x \mathcal{I}_{q i}\left(\frac{\vec{b}_{\perp}}{x}, x\right) .
$$

Using eq. (A.1), we find

$$
J_{\mathrm{EEC}}^{q(0)}\left(\vec{b}_{\perp}\right)=1 .
$$

At NLO, we can write the logarithm appearing in the splitting functions as

$$
\ln \left(\frac{\vec{b}_{\perp}^{2} \mu^{2}}{x^{2} b_{0}^{2}}\right)=\ln \left(\frac{\vec{b}_{\perp}^{2} \mu^{2}}{b_{0}^{2}}\right)-\ln \left(x^{2}\right) .
$$

The calculation of logarithmically enhanced terms is then made trivial using the sum rule for the tree level splitting functions

$$
\sum_{i} \int_{0}^{1} d x x P_{i j}^{(0)}(x)=0 .
$$

However, the splitting functions enter the calculation of the constant, and we find

$$
\begin{aligned}
c_{1}^{J} & =\int_{0}^{1} d x x C_{F}\left[\ln \left(x^{2}\right) P_{q q}^{(0)}(x)+2(1-x)\right]+\int_{0}^{1} d x x C_{F}\left[\ln \left(x^{2}\right) P_{q g}^{(0)}(x)+2 x\right] \\
& =\left(4-8 \zeta_{2}\right) C_{F} .
\end{aligned}
$$

We therefore find that the one-loop jet function for the EEC is given by

$$
J_{\mathrm{EEC}}^{q(1)}\left(\vec{b}_{\perp}\right)=C_{F}\left(-2 L_{\perp} L_{Q}+3 L_{\perp}\right)+c_{1}^{J} .
$$




\section{B Logarithmic structure to NNLO}

In this appendix we perform a check of our factorization formula for the EEC observable by reproducing the known logarithmic structure up to NNLO. We begin by collecting a number of ingredients that will be required, namely the hard, jet and soft functions, their associated anomalous dimensions, and results for vector plus functions that will allow us to treat the integrals appearing in the factorization theorem.

The full scale dependent soft function is given by

$$
\begin{aligned}
S_{\mathrm{EEC}}\left(\vec{b}_{\perp}, \mu, \nu\right)= & \exp \left\{\left(\frac{\alpha_{s}}{4 \pi}\right)\left[c_{1}^{\mathrm{EEC}}+\frac{1}{2} \Gamma_{0}^{\mathrm{cusp}} L_{\perp}^{2}+\gamma_{0}^{r} L_{r}-L_{\perp}\left(\gamma_{0}^{s}+\Gamma_{0}^{\mathrm{cusp}} L_{r}\right)\right]\right. \\
& +\left(\frac{\alpha_{s}}{4 \pi}\right)^{2}\left[c_{2}^{\mathrm{EEC}}+\gamma_{1}^{r} L_{r}+\frac{1}{6} \Gamma_{0}^{\mathrm{cusp}} L_{\perp}^{3} \beta_{0}+L_{\perp}^{2}\left(\frac{\Gamma_{1}^{\text {cusp }}}{2}-\frac{\gamma_{0}^{s} \beta_{0}}{2}-\frac{1}{2} \Gamma_{0}^{\mathrm{cusp}} L_{r} \beta_{0}\right)\right. \\
& \left.+L_{\perp}\left(-\gamma_{1}^{s}+c_{1}^{\perp} \beta_{0}+L_{r}\left(-\Gamma_{1}^{\mathrm{cusp}}+\gamma_{0}^{r} \beta_{0}\right)\right)\right] \\
& +\left(\frac{\alpha_{s}}{4 \pi}\right)^{3}\left[c_{3}^{\mathrm{EEC}}+\gamma_{2}^{r} L_{r}+\frac{1}{12} \Gamma_{0}^{\text {cusp }} L_{\perp}^{4} \beta_{0}^{2}\right. \\
& +L_{\perp}^{3}\left(\frac{\Gamma_{1}^{\text {cusp }} \beta_{0}}{3}+\frac{1}{3}-\gamma_{0}^{s} \beta_{0}^{2}-\frac{1}{3} \Gamma_{0}^{\text {cusp }} L_{r} \beta_{0}^{2}+\frac{\Gamma_{0}^{\text {cusp }} \beta_{1}}{6}\right) \\
& +L_{\perp}^{2}\left(\frac{\Gamma_{2}^{\text {cusp }}}{2}-\gamma_{1}^{s} \beta_{0}+c_{1}^{\perp} \beta_{0}^{2}-\frac{\gamma_{0}^{s} \beta_{1}}{2}+L_{r}\left(-\Gamma_{1}^{\text {cusp }} \beta_{0}+\gamma_{0}^{r} \beta_{0}^{2}-\frac{\Gamma_{0}^{\text {cusp }} \beta_{1}}{2}\right)\right) \\
& \left.\left.+L_{\perp}\left(-\gamma_{2}^{s}+2 c_{2}^{\perp} \beta_{0}+c_{1}^{\perp} \beta_{1}+L_{r}\left(-\Gamma_{2}^{\text {cusp }}+2 \gamma_{1}^{r} \beta_{0}+\gamma_{0}^{r} \beta_{1}\right)\right)\right]+\mathcal{O}\left(\alpha_{s}^{4}\right)\right\}
\end{aligned}
$$

where $L_{r}=\ln \left(\nu^{2} \vec{b}_{\perp}^{2} / b_{0}^{2}\right)$ is the rapidity logarithm, and $L_{\perp}=\ln \left(\vec{b}_{\perp}^{2} \mu^{2} / b_{0}^{2}\right)$, as before. Expanded to $\mathcal{O}\left(\alpha_{s}^{2}\right)$, as is relevant for our check to NNLO, we find

$$
\begin{aligned}
S_{\mathrm{EEC}}\left(\vec{b}_{\perp}, \mu, \nu\right)= & +\left(\frac{\alpha_{s}}{4 \pi}\right)\left[c_{1}^{\mathrm{EEC}}+\frac{1}{2} \Gamma_{0}^{\mathrm{cusp}} L_{\perp}^{2}+\gamma_{0}^{r} L_{r}+L_{\perp}\left(-\gamma_{0}^{s}-\Gamma_{0}^{\mathrm{cusp}} L_{r}\right)\right] \\
& +\left(\frac{\alpha_{s}}{4 \pi}\right)^{2}\left[c_{2}^{\mathrm{EEC}}+\gamma_{1}^{r} L_{r}+\frac{1}{6} \Gamma_{0}^{\mathrm{cusp}} L_{\perp}^{3} \beta_{0}+L_{\perp}^{2}\left(\frac{\Gamma_{1}^{\mathrm{cusp}}}{2}-\frac{\gamma_{0}^{s} \beta_{0}}{2}-\frac{1}{2} \Gamma_{0}^{\mathrm{cusp}} L_{r} \beta_{0}\right)\right. \\
& \left.+L_{\perp}\left(-\gamma_{1}^{s}+c_{1}^{\mathrm{EEC}} \beta_{0}+L_{r}\left(-\Gamma_{1}^{\mathrm{cusp}}+\gamma_{0}^{r} \beta_{0}\right)\right)\right] \\
& +\frac{1}{2}\left(\frac{\alpha_{s}}{4 \pi}\right)^{2}\left[c_{1}^{\mathrm{EEC}}+\frac{1}{2} \Gamma_{0}^{\mathrm{cusp}} L_{\perp}^{2}+\gamma_{0}^{r} L_{r}-L_{\perp}\left(\gamma_{0}^{s}+\Gamma_{0}^{\mathrm{cusp}} L_{r}\right)\right]^{2}+\mathcal{O}\left(\alpha_{s}^{3}\right) .(\mathrm{B} .2)
\end{aligned}
$$

We will normalize the hard function so that its tree level value is 1 , by pulling out the tree level total cross section

$$
\sigma_{0}=\frac{4 \pi \alpha^{2}}{Q^{2}} \sum_{q} \sigma_{q} e_{q}^{2}
$$


The scale dependent hard function is then given to two-loops by (see e.g. [26])

$$
\begin{aligned}
H= & +\left(\frac{\alpha_{s}}{4 \pi}\right)\left(-\frac{1}{2} \Gamma_{0}^{\text {cusp }} L_{H}^{2}+\gamma_{0}^{H} L_{H}+c_{1}^{H}\right) \\
& +\left(\frac{\alpha_{s}}{4 \pi}\right)^{2}\left[\frac{1}{8}\left(\Gamma_{0}^{\text {cusp }}\right)^{2} L_{H}^{4}-\left(\frac{\beta_{0} \Gamma_{0}^{\text {cusp }}}{6}+\frac{\gamma_{0}^{H} \Gamma_{0}^{\text {cusp }}}{2}\right) L_{H}^{3}\right. \\
& \left.+\left(\frac{\left(\gamma_{0}^{H}\right)^{2}}{2}+\frac{\beta_{0} \gamma_{0}^{H}}{2}-\frac{\Gamma_{1}^{\text {cusp }}}{2}\right) L_{H}^{2}+\gamma_{1}^{H} L_{H}+c_{1}^{H}\left(-\frac{\Gamma_{0}^{\text {cusp }}}{2} L_{H}^{2}+L_{H}\left(\beta_{0}+\gamma_{0}^{H}\right)\right)+c_{2}^{H}\right] \\
& +\mathcal{O}\left(\alpha_{s}^{3}\right),
\end{aligned}
$$

which is sufficient for our purposes. Here $L_{H}=\ln \frac{\mu^{2}}{Q^{2}}$, and the hard function constants are given by

$$
\begin{aligned}
c_{1}^{H}= & C_{F}\left(14 \zeta_{2}-16\right), \\
c_{2}^{H}= & C_{A} C_{F}\left(\frac{1061 \zeta_{2}}{9}+\frac{626 \zeta_{3}}{9}-16 \zeta_{4}-\frac{51157}{324}\right)+C_{F}^{2}\left(-166 \zeta_{2}-60 \zeta_{3}+201 \zeta_{4}+\frac{511}{4}\right) \\
& +C_{F} n_{f}\left(-\frac{182 \zeta_{2}}{9}+\frac{4 \zeta_{3}}{9}+\frac{4085}{162}\right) .
\end{aligned}
$$

The hard anomalous dimensions are given by

$$
\begin{aligned}
& \gamma_{0}^{H}=-6 C_{F}, \\
& \gamma_{1}^{H}=C_{F}^{2}\left(-3+24 \zeta_{2}-48 \zeta_{3}\right)+C_{F} C_{A}\left(-\frac{961}{27}-22 \zeta_{2}+52 \zeta_{3}\right)+C_{F} T_{F} n_{f}\left(\frac{260}{27}+8 \zeta_{2}\right) .
\end{aligned}
$$

We will also need the one-loop running of $\alpha_{s}$, which is given by

$$
\alpha_{s}(\mu)=\alpha_{s}\left(\mu_{R}\right)\left(1-\frac{\alpha_{s}\left(\mu_{R}\right)}{4 \pi} \beta_{0} \ln \left(\frac{\mu^{2}}{\mu_{R}^{2}}\right)+\mathcal{O}\left(\alpha_{s}^{2}\right)\right),
$$

where

$$
\beta_{0}=\frac{11}{3} C_{A}-\frac{2}{3} n_{f}
$$

The quark cusp anomalous dimensions are [101]

$$
\begin{aligned}
& \Gamma_{0}^{\text {cusp }}=4 C_{F}, \\
& \Gamma_{1}^{\text {cusp }}=C_{A} C_{F}\left(\frac{268}{9}-8 \zeta_{2}\right)-C_{F} n_{f} \frac{40}{9} .
\end{aligned}
$$

Since we have set up our factorization as a marginalization over $\vec{k}_{\perp}$, at intermediate stages of our calculation we will encounter vector plus distributions. Definitions of vector plus distributions can be found in [25, 135]. In particular, we will use the logarithmic plus distributions

$$
\mathcal{L}_{n}\left(\vec{p}_{\perp}, \mu\right) \equiv \frac{1}{\pi \mu^{2}}\left[\frac{\mu^{2}}{\vec{p}_{\perp}^{2}} \ln ^{n} \frac{\vec{p}_{\perp}^{2}}{\mu^{2}}\right]_{+}^{\mu} .
$$


Since we will be interested in extracting the fixed order expansion of our resummed result, we will choose particular $\mu$ and $\nu$ scales. After having done this, all logarithms will appear in the form

$$
L_{b}^{n} \equiv \ln ^{n}\left(\frac{\vec{b}_{\perp}^{2} Q^{2} e^{2 \gamma_{E}}}{4}\right) .
$$

Relevant results for Fourier transforms of logarithms can be found in [135]. Here we will explicitly need

$$
\begin{aligned}
\mathrm{FT}^{-1}[1] & =\delta\left(\vec{p}_{\perp}\right), \\
\mathrm{FT}^{-1}\left[L_{b}\right] & =-\mathcal{L}_{0}\left(\vec{p}_{\perp}, Q\right), \\
\mathrm{FT}^{-1}\left[L_{b}^{2}\right] & =2 \mathcal{L}_{1}\left(\vec{p}_{\perp}, Q\right), \\
\mathrm{FT}^{-1}\left[L_{b}^{3}\right] & =-3 \mathcal{L}_{2}\left(\vec{p}_{\perp}, Q\right)-4 \zeta_{3} \delta\left(\vec{p}_{\perp}\right), \\
\mathrm{FT}^{-1}\left[L_{b}^{4}\right] & =4 \mathcal{L}_{3}\left(\vec{p}_{\perp}, Q\right)+16 \zeta_{3} \mathcal{L}_{0}\left(\vec{p}_{\perp}, Q\right) .
\end{aligned}
$$

We can now show that our result reproduces the known leading power results for the EEC observable. We will expand the cross section perturbatively as

$$
\frac{1}{\sigma_{0}} \frac{d \sigma}{d z}=\frac{d \sigma^{(0)}}{d z}+\frac{d \sigma^{(1)}}{d z}+\frac{d \sigma^{(2)}}{d z}+\cdots,
$$

where the superscript indicates the perturbative order. At LO and in the back-to-back region, we have

$$
\begin{aligned}
\frac{d \sigma^{(0)}}{d z} & =\frac{1}{2} H^{(0)}(Q) \int d^{2} \vec{k}_{\perp} \int \frac{d^{2} \vec{b}_{\perp}}{(2 \pi)^{2}} e^{-i \vec{b}_{\perp} \cdot \vec{k}_{\perp}} \delta\left(1-z-\frac{\vec{k}_{\perp}^{2}}{Q^{2}}\right) \\
& =\frac{1}{2} H^{(0)}(Q) \delta(1-z) .
\end{aligned}
$$

Note that we have ignored the collinear region, which gives a $\frac{1}{2} \delta(z)$ at LO. To reproduce the NLO and NNLO fixed order results, we choose to evaluate everything at the jet scale

$$
\mu_{J}^{2}=\frac{b_{0}^{2}}{\vec{b}_{\perp}^{2}}, \quad \nu_{J}=Q .
$$

This is convenient, since this is the natural $\mu$ scale for both the soft and jet functions. We then have

$$
L_{H} \rightarrow-L_{b}, \quad L_{r} \rightarrow L_{b}, \quad L_{\perp} \rightarrow 0 .
$$

This considerably simplifies the expression for the soft function

$$
\begin{aligned}
S_{\mathrm{EEC}}\left(\vec{b}_{\perp}, \mu^{2}=\frac{b_{0}^{2}}{\vec{b}_{\perp}^{2}}, \nu=Q\right)= & +\left(\frac{\alpha_{s}\left(\mu_{J}\right)}{4 \pi}\right)\left[c_{1}^{\mathrm{EEC}}+\gamma_{0}^{r} L_{b}\right]+\left(\frac{\alpha_{s}\left(\mu_{J}\right)}{4 \pi}\right)^{2}\left[c_{2}^{\mathrm{EEC}}+\gamma_{1}^{r} L_{b}\right] \\
& +\frac{1}{2}\left(\frac{\alpha_{s}\left(\mu_{J}\right)}{4 \pi}\right)^{2}\left[c_{1}^{\mathrm{EEC}}+\gamma_{0}^{r} L_{b}\right]^{2}+\mathcal{O}\left(\alpha_{s}^{3}\right) .
\end{aligned}
$$


For the hard function, we have

$$
\begin{aligned}
H\left(\mu^{2}=\frac{b_{0}^{2}}{\vec{b}_{\perp}^{2}}\right)= & 1+\left(\frac{\alpha_{s}\left(\mu_{J}\right)}{4 \pi}\right)\left(-\frac{1}{2} \Gamma_{0}^{\text {cusp }} L_{b}^{2}-\gamma_{0}^{H} L_{b}+c_{1}^{H}\right) \\
& +\left(\frac{\alpha_{s}\left(\mu_{J}\right)}{4 \pi}\right)^{2}\left[\frac{1}{8}\left(\Gamma_{0}^{\text {cusp }}\right)^{2} L_{b}^{4}+\left(\frac{\beta_{0} \Gamma_{0}^{\text {cusp }}}{6}+\frac{\gamma_{0}^{H} \Gamma_{0}^{\text {cusp }}}{2}\right) L_{b}^{3}\right. \\
& \left.+\left(\frac{\left(\gamma_{0}^{H}\right)^{2}}{2}+\frac{\beta_{0} \gamma_{0}^{H}}{2}-\frac{\Gamma_{1}^{\text {cusp }}}{2}\right) L_{b}^{2}-\gamma_{1}^{H} L_{b}+c_{1}^{H}\left(-\frac{\Gamma_{0}^{\text {cusp }}}{2} L_{b}^{2}-L_{b}\left(\beta_{0}+\gamma_{0}^{H}\right)\right)+c_{2}^{H}\right] \\
& +\mathcal{O}\left(\alpha_{s}^{3}\right) .
\end{aligned}
$$

Finally, the one-loop constant for the jet function is

$$
J_{\mathrm{EEC}}^{q}\left(\vec{b}_{\perp}, \mu^{2}=\frac{b_{0}^{2}}{\vec{b}_{\perp}^{2}}, \nu=Q\right)=1+\left(\frac{\alpha_{s}\left(\mu_{J}\right)}{4 \pi}\right) c_{1}^{J}+\mathcal{O}\left(\alpha_{s}^{2}\right)
$$

where the term at $\mathcal{O}\left(\alpha_{s}^{2}\right)$ is purely a constant with no logarithmic dependence, due to the choice of scales.

At NLO, we find

$$
\begin{aligned}
\frac{d \sigma^{(1)}}{d z} & =\frac{1}{2} H^{(0)}(Q)\left(\frac{\alpha_{s}}{4 \pi}\right) \int d^{2} \vec{k}_{\perp} \int \frac{d^{2} \vec{b}_{\perp}}{(2 \pi)^{2}} e^{-i \vec{b}_{\perp} \cdot \vec{k}_{\perp}}\left[\left(c_{1}^{H}+2 c_{1}^{J}+c_{1}^{\text {EEC }}\right)-\gamma_{0}^{H} L_{b}-\frac{1}{2} \Gamma_{0}^{\text {cusp }} L_{b}^{2}\right] \delta\left(1-z-\frac{\vec{k}_{\perp}^{2}}{Q^{2}}\right) \\
& =\frac{1}{2} H^{(0)}(Q)\left(\frac{\alpha_{s}}{4 \pi}\right) \int d^{2} \vec{k}_{\perp} \int \frac{d^{2} \vec{b}_{\perp}}{(2 \pi)^{2}} e^{-i \vec{b}_{\perp} \cdot \vec{k}_{\perp}} C_{F}\left[\left(-4 \zeta_{2}-8\right)+6 L_{b}-2 L_{b}^{2}\right] \delta\left(1-z-\frac{\vec{k}_{\perp}^{2}}{Q^{2}}\right) \\
& =\frac{1}{2} H^{(0)}(Q)\left(\frac{\alpha_{s}}{4 \pi}\right) C_{F} \int d^{2} \vec{k}_{\perp}\left[\left(-4 \zeta_{2}-8\right) \delta^{(2)}\left(\vec{k}_{\perp}\right)-6 \mathcal{L}_{0}\left(\vec{k}_{\perp}, \mu\right)-4 \mathcal{L}_{1}\left(\vec{k}_{\perp}, \mu\right)\right] \delta\left(1-z-\frac{\vec{k}_{\perp}^{2}}{Q^{2}}\right) \\
& =H^{(0)}(Q)\left(\frac{\alpha_{s}}{4 \pi}\right)\left(C_{F}\left(-2 \zeta_{2}-4\right) \delta(1-z)-3 C_{F}\left[\frac{1}{1-z}\right]_{+}-2 C_{F}\left[\frac{\ln (1-z)}{1-z}\right]_{+}\right) .
\end{aligned}
$$

To perform the final integral over the $\vec{k}_{\perp}$ appearing in the factorization theorem, we used

$$
\begin{aligned}
\int d^{2} \vec{k}_{\perp} \mathcal{L}_{n}\left(\vec{k}_{\perp}, Q\right) \delta & \left(1-z-\frac{\vec{k}_{\perp}^{2}}{Q^{2}}\right) \\
= & \pi Q^{2} \int d\left|\vec{k}_{\perp}\right| \delta\left(\left|\vec{k}_{\perp}\right|-Q \sqrt{1-z}\right) \mathcal{L}_{n}\left(\vec{k}_{\perp}, Q\right)=\mathcal{L}_{n}(1-z)
\end{aligned}
$$

where

$$
\mathcal{L}_{n}(1-z)=\left[\frac{\ln (1-z)}{1-z}\right]_{+},
$$

is the standard one-dimensional logarithmic plus distribution.

For the NNLO result, we provide slightly more details of the calculation. We begin by expanding the result in impact parameter space, keeping only the logarithmic terms. 
We find

$$
\begin{aligned}
& \left.H\left(\mu^{2}=\frac{b_{0}^{2}}{\vec{b}_{\perp}^{2}}\right)\left[J_{\mathrm{EEC}}^{q}\left(\vec{b}_{\perp}, \mu^{2}=\frac{b_{0}^{2}}{\vec{b}_{\perp}^{2}}, \nu=Q\right)\right]^{2} S_{\mathrm{EEC}}\left(\vec{b}_{\perp}, \mu^{2}=\frac{b_{0}^{2}}{\vec{b}_{\perp}^{2}}, \nu=Q\right)\right|_{\alpha_{s}^{2}} \\
& =\left(\frac{\alpha_{s}\left(\mu_{J}\right)}{4 \pi}\right)^{2} \frac{1}{8}\left(\Gamma_{0}^{\text {cusp }}\right)^{2} L_{b}^{4} \\
& +\left(\frac{\alpha_{s}\left(\mu_{J}\right)}{4 \pi}\right)^{2}\left[\left(\frac{\beta_{0} \Gamma_{0}^{\text {cusp }}}{6}+\frac{\gamma_{0}^{H} \Gamma_{0}^{\text {cusp }}}{2}\right)-\frac{1}{2} \Gamma_{0}^{\text {cusp }} \gamma_{0}^{r}\right] L_{b}^{3} \\
& +\left(\frac{\alpha_{s}\left(\mu_{J}\right)}{4 \pi}\right)^{2}\left[\left(\frac{\left(\gamma_{0}^{r}\right)^{2}}{2}+\frac{\left(\gamma_{0}^{H}\right)^{2}}{2}+\frac{\beta_{0} \gamma_{0}^{H}}{2}-\left(\frac{\Gamma_{1}^{\text {cusp }}}{2}\right)\right)+\left(c_{1}^{\mathrm{EEC}}+2 c_{1}^{J}+c_{1}^{H}\right)\left(-\frac{\Gamma_{0}^{\text {cusp }}}{2}\right)-\gamma_{0}^{H} \gamma_{0}^{r}\right] L_{b}^{2} \\
& +\left(\frac{\alpha_{s}\left(\mu_{J}\right)}{4 \pi}\right)^{2}\left[\gamma_{1}^{r}-\gamma_{1}^{H}-c_{1}^{H}\left(\beta_{0}+\gamma_{0}^{H}\right)+2 c_{1}^{J} \gamma_{0}^{r}-2 c_{1}^{J} \gamma_{0}^{H}-\gamma_{0}^{H} c_{1}^{\mathrm{EEC}}+\gamma_{0}^{r} c_{1}^{H}\right] L_{b} .
\end{aligned}
$$

In the literature, it is conventional to write the above expression evaluated with $\alpha_{s}$ at the hard scale, $Q$, which can be done using

$$
\alpha_{s}\left(\mu_{J}\right)=\alpha_{s}(Q)\left(1+\frac{\alpha_{s}(Q)}{4 \pi} \beta_{0} L_{b}\right) .
$$

This modifies the NNLO result by $\beta_{0}$ terms multiplying the NLO result, namely by

$$
\left(\frac{\alpha_{s}(Q)}{4 \pi}\right)^{2}\left[-\frac{1}{2} \Gamma_{0}^{\mathrm{cusp}} \beta_{0} L_{b}^{3}-\gamma_{0}^{H} \beta_{0} L_{b}^{2}+\left(2 c_{1}^{J}+c_{1}^{H}+c_{1}^{\mathrm{EEC}}\right) \beta_{0} L_{b}\right] .
$$

Written with $\alpha_{s}$ at the hard scale, we then have

$$
\begin{aligned}
H & \cdot\left(J_{\mathrm{EEC}}^{q}\left(\vec{b}_{\perp}\right)\right)^{2} \cdot S_{\mathrm{EEC}}\left(\vec{b}_{\perp}\right)=\left(\frac{\alpha_{s}(Q)}{4 \pi}\right)^{2} \frac{1}{8}\left(\Gamma_{0}^{\mathrm{cusp}}\right)^{2} L_{b}^{4} \\
& +\left(\frac{\alpha_{s}(Q)}{4 \pi}\right)^{2}\left[-\frac{\beta_{0}}{3}+\frac{\gamma_{0}^{H}}{2}-\frac{\gamma_{0}^{r}}{2}\right] \Gamma_{0}^{\mathrm{cusp}} L_{b}^{3} \\
& +\left(\frac{\alpha_{s}(Q)}{4 \pi}\right)^{2}\left[\frac{\left(\gamma_{0}^{r}\right)^{2}}{2}+\frac{\left(\gamma_{0}^{H}\right)^{2}}{2}-\frac{\beta_{0} \gamma_{0}^{H}}{2}-\frac{\Gamma_{1}^{\mathrm{cusp}}}{2}-\gamma_{0}^{H} \gamma_{0}^{r}-\frac{\Gamma_{0}^{\mathrm{cusp}}}{2}\left(c_{1}^{\mathrm{EEC}}+2 c_{1}^{J}+c_{1}^{H}\right)\right] L_{b}^{2} \\
& +\left(\frac{\alpha_{s}(Q)}{4 \pi}\right)^{2}\left[\gamma_{1}^{r}-\gamma_{1}^{H}-c_{1}^{H} \gamma_{0}^{H}+2 c_{1}^{J} \gamma_{0}^{r}-2 c_{1}^{J} \gamma_{0}^{H}-\gamma_{0}^{H} c_{1}^{\mathrm{EEC}}+\gamma_{0}^{r} c_{1}^{H}+\left(2 c_{1}^{J}+c_{1}^{\mathrm{EEC}}\right) \beta_{0}\right] L_{b} .
\end{aligned}
$$

Performing the Fourier transform, we find

$$
\begin{aligned}
& \int d^{2} \vec{b}_{\perp} e^{-i \vec{b}_{\perp} \cdot \vec{k}_{\perp}} H \cdot\left(J_{\mathrm{EEC}}^{q}\left(\vec{b}_{\perp}\right)\right)^{2} \cdot S_{\mathrm{EEC}}\left(\vec{b}_{\perp}\right)= \\
& \left(\frac{\alpha_{s}(Q)}{4 \pi}\right)^{2} \frac{1}{8}\left(\Gamma_{0}^{\mathrm{cusp}}\right)^{2}\left(4 \mathcal{L}_{3}\left(\vec{k}_{\perp}, Q\right)+16 \zeta_{3} \mathcal{L}_{0}\left(\vec{k}_{\perp}, Q\right)\right) \\
& +\left(\frac{\alpha_{s}(Q)}{4 \pi}\right)^{2}\left[-\frac{\beta_{0}}{3}+\frac{\gamma_{0}^{H}}{2}-\frac{\gamma_{0}^{r}}{2}\right] \Gamma_{0}^{\mathrm{cusp}}\left(-3 \mathcal{L}_{2}\left(\vec{k}_{\perp}, Q\right)\right) \\
& +\left(\frac{\alpha_{s}(Q)}{4 \pi}\right)^{2}\left[\frac{\left(\gamma_{0}^{r}\right)^{2}}{2}+\frac{\left(\gamma_{0}^{H}\right)^{2}}{2}-\frac{\beta_{0} \gamma_{0}^{H}}{2}-\frac{\Gamma_{1}^{\mathrm{cusp}}}{2}-\gamma_{0}^{H} \gamma_{0}^{r}-\frac{\Gamma_{0}^{\text {cusp }}}{2}\left(c_{1}^{\mathrm{EEC}}+2 c_{1}^{J}+c_{1}^{H}\right)\right]\left(2 \mathcal{L}_{1}\left(\vec{k}_{\perp}, Q\right)\right) \\
& +\left(\frac{\alpha_{s}(Q)}{4 \pi}\right)^{2}\left[\gamma_{1}^{r}-\gamma_{1}^{H}-c_{1}^{H} \gamma_{0}^{H}+2 c_{1}^{J} \gamma_{0}^{r}-2 c_{1}^{J} \gamma_{0}^{H}-\gamma_{0}^{H} c_{1}^{\mathrm{EEC}}+\gamma_{0}^{r} c_{1}^{H}+\left(2 c_{1}^{J}+c_{1}^{\mathrm{EEC}}\right) \beta_{0}\right]\left(-\mathcal{L}_{0}\left(\vec{k}_{\perp}, Q\right)\right) .
\end{aligned}
$$


This allows us to immediately write down the final result for the cross section in terms of the $z$ variable

$$
\begin{aligned}
\frac{d \sigma^{(2)}}{d z} & =\frac{1}{2}\left(\frac{\alpha_{s}(Q)}{4 \pi}\right)^{2} \frac{1}{2}\left(\Gamma_{0}^{\mathrm{cusp}}\right)^{2} \mathcal{L}_{3}(1-z) \\
+ & \frac{1}{2}\left(\frac{\alpha_{s}(Q)}{4 \pi}\right)^{2}\left[\beta_{0}-\frac{3 \gamma_{0}^{H}}{2}+\frac{3 \gamma_{0}^{r}}{2}\right] \Gamma_{0}^{\mathrm{cusp}} \mathcal{L}_{2}(1-z) \\
+ & \frac{1}{2}\left(\frac{\alpha_{s}(Q)}{4 \pi}\right)^{2}\left[\left(\gamma_{0}^{r}\right)^{2}+\left(\gamma_{0}^{H}\right)^{2}-\beta_{0} \gamma_{0}^{H}-\Gamma_{1}^{\mathrm{cusp}}-2 \gamma_{0}^{H} \gamma_{0}^{r}-\Gamma_{0}^{\mathrm{cusp}}\left(c_{1}^{\mathrm{EEC}}+2 c_{1}^{J}+c_{1}^{H}\right)\right] \mathcal{L}_{1}(1-z) \\
+ & \frac{1}{2}\left(\frac{\alpha_{s}(Q)}{4 \pi}\right)^{2}\left[2\left(\Gamma_{0}^{\mathrm{cusp}}\right)^{2} \zeta_{3}-\gamma_{1}^{r}+\gamma_{1}^{H}\right. \\
& \left.+c_{1}^{H} \gamma_{0}^{H}-2 c_{1}^{J} \gamma_{0}^{r}+2 c_{1}^{J} \gamma_{0}^{H}+\gamma_{0}^{H} c_{1}^{\mathrm{EEC}}-\gamma_{0}^{r} c_{1}^{H}-\left(2 c_{1}^{J}+c_{1}^{\mathrm{EEC}}\right) \beta_{0}\right] \mathcal{L}_{0}(1-z) .
\end{aligned}
$$

Plugging in the values of the different anomalous dimensions, we have

$$
\begin{aligned}
& \frac{d \sigma^{(2)}}{d z}=\left(\frac{\alpha_{s}(Q)}{4 \pi}\right)^{2} 4 C_{F}^{2} \mathcal{L}_{3}(1-z) \\
& +\left(\frac{\alpha_{s}(Q)}{4 \pi}\right)^{2}\left[18 C_{F}^{2}+\frac{22}{3} C_{A} C_{F}-\frac{4}{3} n_{f} C_{F}\right] \mathcal{L}_{2}(1-z) \\
& +\left(\frac{\alpha_{s}(Q)}{4 \pi}\right)^{2}\left[C_{F} C_{A}\left(4 \zeta_{2}-\frac{35}{9}\right)+\frac{2}{9} C_{F} n_{f}+C_{F}^{2}\left(8 \zeta_{2}+34\right)\right] \mathcal{L}_{1}(1-z) \\
& +\left(\frac{\alpha_{s}(Q)}{4 \pi}\right)^{2}\left[\left(\frac{45}{2}+24 \zeta_{2}-8 \zeta_{3}\right) C_{F}^{2}+\left(-\frac{35}{2}+22 \zeta_{2}+12 \zeta_{3}\right) C_{F} C_{A}+\left(3-4 \zeta_{2}\right) C_{F} n_{f}\right] \mathcal{L}_{0}(1-z) .
\end{aligned}
$$

We can compare this result to a previous NNLL result in the literature [10], computed in the CSS formalism. The result of [10] was written as

$$
\begin{aligned}
\frac{1}{\sigma_{T}} \frac{d \sigma}{d \cos \chi}= & \frac{1}{4 y} \frac{\alpha_{s}(Q)}{\pi}\left[-A^{(1)} \ln y+B^{(1)}\right] \\
+\frac{1}{4 y}\left(\frac{\alpha_{s}(Q)}{\pi}\right)^{2}[ & \frac{1}{2}\left(A^{(1)}\right)^{2} \ln ^{3} y+\left(-\frac{3}{2} B^{(1)} A^{(1)}+\frac{\beta_{0}}{4} A^{(1)}\right) \ln ^{2} y \\
& +\left(-A^{(2)}-\frac{\beta_{0}}{4} B^{(1)}+\left(B^{(1)}\right)^{2}-A^{(1)} H^{(1)}\right) \ln y \\
& \left.+B^{(2)}+B^{(1)} H^{(1)}+2 \zeta_{3}\left(A^{(1)}\right)^{2}\right]
\end{aligned}
$$

where $y=\sin ^{2}(\pi-\chi) / 2=1-z$. The required constants appearing in eq. (B.37) are given by

$$
\begin{aligned}
A^{(1)} & =\frac{\Gamma_{0}^{\text {cusp }}}{4}, & A^{(2)} & =\frac{\Gamma_{1}^{\text {cusp }}}{16}, \\
B^{(1)} & =-\frac{3}{2} C_{F}, & B^{(2)} & =-\frac{1}{2} \gamma_{q}^{(2)}+C_{F} \frac{\beta_{0}}{4}\left(5 \zeta_{2}-2\right), \\
H^{(1)} & =-C_{F}\left(\frac{11}{4}+\zeta_{2}\right), & &
\end{aligned}
$$

and

$$
\gamma_{q}^{(2)}=C_{F}^{2}\left(\frac{3}{8}-3 \zeta_{2}+6 \zeta_{3}\right)+C_{F} C_{A}\left(\frac{17}{24}+\frac{11}{3} \zeta_{2}-3 \zeta_{3}\right)-C_{F} n_{f} T_{R}\left(\frac{1}{6}+\frac{4}{3} \zeta_{2}\right) .
$$


Note that to perform the comparison, one must take into account that the formula of eq. (B.37) from [10] normalizes to the NLO total cross section

$$
\sigma_{T}=\sigma_{0}\left(1+3\left(\frac{\alpha_{s}(Q)}{4 \pi}\right) C_{F}\right)
$$

while in eq. (B.35), we have normalized only to $\sigma_{0}$. We find exact agreement with their result. The result of eq. (B.37) was verified by comparison with the fixed order program EvEnT2 [157, 158], and was shown to correctly reproduce the logarithmic structure to this order. This provides a highly non-trivial check of our factorization theorem. In particular, the difference found in [10] between the $B^{(2)}$ coefficient for the EEC and $B_{q, \mathrm{NS}}^{(2)}$ (see equations 19 and 21 in [10]) is naturally reproduced by our factorization theorem.

Open Access. This article is distributed under the terms of the Creative Commons Attribution License (CC-BY 4.0), which permits any use, distribution and reproduction in any medium, provided the original author(s) and source are credited.

\section{References}

[1] A. Gehrmann-De Ridder, T. Gehrmann, E.W.N. Glover and G. Heinrich, NNLO corrections to event shapes in $e^{+} e^{-}$annihilation, JHEP 12 (2007) 094 [arXiv:0711.4711] [INSPIRE].

[2] A. Gehrmann-De Ridder, T. Gehrmann, E.W.N. Glover and G. Heinrich, Second-order QCD corrections to the thrust distribution, Phys. Rev. Lett. 99 (2007) 132002 [arXiv: 0707.1285] [INSPIRE].

[3] S. Weinzierl, NNLO corrections to 3-jet observables in electron-positron annihilation, Phys. Rev. Lett. 101 (2008) 162001 [arXiv:0807.3241] [INSPIRE].

[4] S. Weinzierl, Event shapes and jet rates in electron-positron annihilation at NNLO, JHEP 06 (2009) 041 [arXiv:0904.1077] [INSPIRE].

[5] S. Catani, L. Trentadue, G. Turnock and B.R. Webber, Resummation of large logarithms in $e^{+} e^{-}$event shape distributions, Nucl. Phys. B 407 (1993) 3 [inSPIRE].

[6] V. Del Duca, C. Duhr, A. Kardos, G. Somogyi and Z. Trócsányi, Three-Jet Production in Electron-Positron Collisions at Next-to-Next-to-Leading Order Accuracy, Phys. Rev. Lett. 117 (2016) 152004 [arXiv: 1603.08927] [INSPIRE].

[7] V. Del Duca et al., Jet production in the CoLoRFulNNLO method: event shapes in electron-positron collisions, Phys. Rev. D 94 (2016) 074019 [arXiv:1606.03453] [INSPIRE].

[8] G. Somogyi and Z. Trócsányi, A Subtraction scheme for computing QCD jet cross sections at NNLO: Regularization of real-virtual emission, JHEP 01 (2007) 052 [hep-ph/0609043] [INSPIRE].

[9] G. Somogyi, Z. Trócsányi and V. Del Duca, A Subtraction scheme for computing QCD jet cross sections at NNLO: Regularization of doubly-real emissions, JHEP 01 (2007) 070 [hep-ph/0609042] [INSPIRE].

[10] D. de Florian and M. Grazzini, The Back-to-back region in $e^{+} e^{-}$energy-energy correlation, Nucl. Phys. B 704 (2005) 387 [hep-ph/0407241] [INSPIRE].

[11] T. Becher and G. Bell, NNLL Resummation for Jet Broadening, JHEP 11 (2012) 126 [arXiv: 1210.0580$]$ [INSPIRE]. 
[12] A. Banfi, H. McAslan, P.F. Monni and G. Zanderighi, The two-jet rate in $e^{+} e^{-}$at next-to-next-to-leading-logarithmic order, Phys. Rev. Lett. 117 (2016) 172001 [arXiv: 1607.03111] [INSPIRE].

[13] C. Frye, A.J. Larkoski, M.D. Schwartz and K. Yan, Precision physics with pile-up insensitive observables, arXiv:1603.06375 [INSPIRE].

[14] C. Frye, A.J. Larkoski, M.D. Schwartz and K. Yan, Factorization for groomed jet substructure beyond the next-to-leading logarithm, JHEP 07 (2016) 064

[arXiv: 1603.09338] [INSPIRE].

[15] J. Mo, F.J. Tackmann and W.J. Waalewijn, A case study of quark-gluon discrimination at NNLL' in comparison to parton showers, Eur. Phys. J. C 77 (2017) 770 [arXiv: 1708.00867] [INSPIRE].

[16] Z. Tulipánt, A. Kardos and G. Somogyi, Energy-energy correlation in electron-positron annihilation at NNLL + NNLO accuracy, Eur. Phys. J. C 77 (2017) 749 [arXiv: 1708.04093] [INSPIRE].

[17] A. Banfi, G.P. Salam and G. Zanderighi, Principles of general final-state resummation and automated implementation, JHEP 03 (2005) 073 [hep-ph/0407286] [INSPIRE].

[18] A. Banfi, H. McAslan, P.F. Monni and G. Zanderighi, A general method for the resummation of event-shape distributions in $e^{+} e^{-}$annihilation, JHEP 05 (2015) 102 [arXiv: 1412.2126] [INSPIRE].

[19] C.W. Bauer, S. Fleming and M.E. Luke, Summing Sudakov logarithms in $B \rightarrow X_{s} \gamma$ in effective field theory, Phys. Rev. D 63 (2000) 014006 [hep-ph/0005275] [INSPIRE].

[20] C.W. Bauer, S. Fleming, D. Pirjol and I.W. Stewart, An Effective field theory for collinear and soft gluons: Heavy to light decays, Phys. Rev. D 63 (2001) 114020 [hep-ph/0011336] [INSPIRE].

[21] C.W. Bauer and I.W. Stewart, Invariant operators in collinear effective theory, Phys. Lett. B 516 (2001) 134 [hep-ph/0107001] [INSPIRE].

[22] C.W. Bauer, D. Pirjol and I.W. Stewart, Soft collinear factorization in effective field theory, Phys. Rev. D 65 (2002) 054022 [hep-ph/0109045] [INSPIRE].

[23] J.-y. Chiu, A. Jain, D. Neill and I.Z. Rothstein, The Rapidity Renormalization Group, Phys. Rev. Lett. 108 (2012) 151601 [arXiv:1104.0881] [INSPIRE].

[24] T. Becher and G. Bell, Analytic Regularization in Soft-Collinear Effective Theory, Phys. Lett. B 713 (2012) 41 [arXiv:1112.3907] [INSPIRE].

[25] J.-Y. Chiu, A. Jain, D. Neill and I.Z. Rothstein, A Formalism for the Systematic Treatment of Rapidity Logarithms in Quantum Field Theory, JHEP 05 (2012) 084 [arXiv:1202.0814] [INSPIRE].

[26] T. Becher and M.D. Schwartz, A precise determination of $\alpha_{s}$ from LEP thrust data using effective field theory, JHEP 07 (2008) 034 [arXiv:0803.0342] [INSPIRE].

[27] R. Abbate, M. Fickinger, A.H. Hoang, V. Mateu and I.W. Stewart, Thrust at $N^{3} L L$ with Power Corrections and a Precision Global Fit for $\alpha_{s}\left(m_{Z}\right)$, Phys. Rev. D 83 (2011) 074021 [arXiv: 1006.3080] [INSPIRE].

[28] A.H. Hoang, D.W. Kolodrubetz, V. Mateu and I.W. Stewart, C-parameter distribution at $N^{3} L L^{\prime}$ including power corrections, Phys. Rev. D 91 (2015) 094017 [arXiv:1411.6633] [INSPIRE]. 
[29] Y.-T. Chien and M.D. Schwartz, Resummation of heavy jet mass and comparison to LEP data, JHEP 08 (2010) 058 [arXiv: 1005.1644] [INSPIRE].

[30] A.H. Hoang, D.W. Kolodrubetz, V. Mateu and I.W. Stewart, Precise determination of $\alpha_{s}$ from the C-parameter distribution, Phys. Rev. D 91 (2015) 094018 [arXiv:1501.04111] [INSPIRE].

[31] P.E.L. Rakow and B.R. Webber, Transverse Momentum Moments of Hadron Distributions in QCD Jets, Nucl. Phys. B 191 (1981) 63 [InSPIRE].

[32] S. Catani, G. Turnock and B.R. Webber, Jet broadening measures in $e^{+} e^{-}$annihilation, Phys. Lett. B 295 (1992) 269 [INSPIRE].

[33] Y.L. Dokshitzer, A. Lucenti, G. Marchesini and G.P. Salam, On the QCD analysis of jet broadening, JHEP 01 (1998) 011 [hep-ph/9801324] [INSPIRE].

[34] Y. Li and H.X. Zhu, Bootstrapping Rapidity Anomalous Dimensions for Transverse-Momentum Resummation, Phys. Rev. Lett. 118 (2017) 022004 [arXiv: 1604.01404] [INSPIRE].

[35] L.J. Dixon, J.M. Drummond and J.M. Henn, Bootstrapping the three-loop hexagon, JHEP 11 (2011) 023 [arXiv: 1108.4461] [INSPIRE].

[36] L.J. Dixon and M. von Hippel, Bootstrapping an NMHV amplitude through three loops, JHEP 10 (2014) 065 [arXiv: 1408.1505] [INSPIRE].

[37] L.J. Dixon, M. von Hippel and A.J. McLeod, The four-loop six-gluon NMHV ratio function, JHEP 01 (2016) 053 [arXiv:1509.08127] [INSPIRE].

[38] S. Caron-Huot, L.J. Dixon, A. McLeod and M. von Hippel, Bootstrapping a Five-Loop Amplitude Using Steinmann Relations, Phys. Rev. Lett. 117 (2016) 241601 [arXiv: 1609.00669] [INSPIRE].

[39] L.J. Dixon, J. Drummond, T. Harrington, A.J. McLeod, G. Papathanasiou and M. Spradlin, Heptagons from the Steinmann Cluster Bootstrap, JHEP 02 (2017) 137 [arXiv: 1612.08976] [INSPIRE].

[40] Y. Li, D. Neill and H.X. Zhu, An Exponential Regulator for Rapidity Divergences, arXiv: 1604.00392 [INSPIRE].

[41] A.J. Larkoski, I. Moult and D. Neill, Toward Multi-Differential Cross Sections: Measuring Two Angularities on a Single Jet, JHEP 09 (2014) 046 [arXiv: 1401.4458] [INSPIRE].

[42] M. Procura, W.J. Waalewijn and L. Zeune, Resummation of Double-Differential Cross Sections and Fully-Unintegrated Parton Distribution Functions, JHEP 02 (2015) 117 [arXiv: 1410.6483] [INSPIRE].

[43] C. Duhr and T. Gehrmann, The two-loop soft current in dimensional regularization, Phys. Lett. B 727 (2013) 452 [arXiv: 1309.4393] [INSPIRE].

[44] Y. Li and H.X. Zhu, Single soft gluon emission at two loops, JHEP 11 (2013) 080 [arXiv: 1309.4391] [INSPIRE].

[45] C. Anastasiou, C. Duhr, F. Dulat and B. Mistlberger, Soft triple-real radiation for Higgs production at N3LO, JHEP 07 (2013) 003 [arXiv: 1302.4379] [INSPIRE].

[46] Y. Li, A. von Manteuffel, R.M. Schabinger and H.X. Zhu, $N^{3} L O$ Higgs boson and Drell-Yan production at threshold: The one-loop two-emission contribution, Phys. Rev. D 90 (2014) 053006 [arXiv:1404.5839] [INSPIRE]. 
[47] H.X. Zhu, On the calculation of soft phase space integral, JHEP 02 (2015) 155 [arXiv:1501.00236] [INSPIRE].

[48] C. Anastasiou, C. Duhr, F. Dulat, E. Furlan, F. Herzog and B. Mistlberger, Soft expansion of double-real-virtual corrections to Higgs production at $N^{3}$ LO, JHEP 08 (2015) 051 [arXiv: 1505.04110] [INSPIRE].

[49] A.A. Vladimirov, Correspondence between Soft and Rapidity Anomalous Dimensions, Phys. Rev. Lett. 118 (2017) 062001 [arXiv:1610.05791] [INSPIRE].

[50] A. Vladimirov, Structure of rapidity divergences in multi-parton scattering soft factors, JHEP 04 (2018) 045 [arXiv: 1707.07606] [INSPIRE].

[51] A.J. Larkoski, D. Neill and J. Thaler, Jet Shapes with the Broadening Axis, JHEP 04 (2014) 017 [arXiv: 1401.2158] [INSPIRE].

[52] A.J. Larkoski and I. Moult, The Singular Behavior of Jet Substructure Observables, Phys. Rev. D 93 (2016) 014017 [arXiv: 1510.08459] [INSPIRE].

[53] J.C. Collins and D.E. Soper, Back-To-Back Jets in QCD, Nucl. Phys. B 193 (1981) 381 [Erratum ibid. B 213 (1983) 545] [INSPIRE].

[54] C.L. Basham, L.S. Brown, S.D. Ellis and S.T. Love, Energy correlations in electron-positron annihilation: testing QCD, Phys. Rev. Lett. 41 (1978) 1585 [INSPIRE].

[55] A. Ali and F. Barreiro, An $O\left(\alpha_{s}\right)^{2}$ Calculation of Energy-energy Correlation in $e^{+} e^{-}$ Annihilation and Comparison With Experimental Data, Phys. Lett. B 118 (1982) 155 [INSPIRE].

[56] S.-C. Chao, D.E. Soper and J.C. Collins, The Order $\alpha_{s}^{2}$ Energy-energy Correlation Function at Small Angles, Nucl. Phys. B 214 (1983) 513 [InSPIRE].

[57] D.G. Richards, W.J. Stirling and S.D. Ellis, Second Order Corrections to the Energy-energy Correlation Function in Quantum Chromodynamics, Phys. Lett. B 119 (1982) 193 [INSPIRE].

[58] S.D. Ellis, D.G. Richards and W.J. Stirling, Fixed Order Perturbation Theory and Leading Logarithms, Phys. Lett. B 136 (1984) 99 [INSPIRE].

[59] H.N. Schneider, G. Kramer and G. Schierholz, Higher Order QCD Corrections to the Energy-energy Correlation Function, Z. Phys. C 22 (1984) 201 [INSPIRE].

[60] D.G. Richards, W.J. Stirling and S.D. Ellis, Energy-energy Correlations to Second Order in Quantum Chromodynamics, Nucl. Phys. B 229 (1983) 317 [InSPIRE].

[61] N.K. Falck and G. Kramer, Theoretical Studies of Energy-energy Correlation in $e^{+} e^{-}$ Annihilation, Z. Phys. C 42 (1989) 459 [InSPIRE].

[62] R. Fiore, A. Quartarolo and L. Trentadue, Energy-energy correlation for $\theta \rightarrow 180^{\circ}$ at LEP, Phys. Lett. B 294 (1992) 431 [InSPIRE].

[63] E.W.N. Glover and M.R. Sutton, The Energy-energy correlation function revisited, Phys. Lett. B 342 (1995) 375 [hep-ph/9410234] [INSPIRE].

[64] K.A. Clay and S.D. Ellis, A Precision calculation of the next-to-leading order energy-energy correlation function, Phys. Rev. Lett. 74 (1995) 4392 [hep-ph/9502223] [INSPIRE].

[65] G. Kramer and H. Spiesberger, A new calculation of the NLO energy-energy correlation function, Z. Phys. C 73 (1997) 495 [hep-ph/9603385] [INSPIRE]. 
[66] Y.L. Dokshitzer, G. Marchesini and B.R. Webber, Nonperturbative effects in the energy-energy correlation, JHEP 07 (1999) 012 [hep-ph/9905339] [INSPIRE].

[67] A.V. Belitsky, S. Hohenegger, G.P. Korchemsky, E. Sokatchev and A. Zhiboedov, From correlation functions to event shapes, Nucl. Phys. B 884 (2014) 305 [arXiv:1309.0769] [INSPIRE].

[68] A.V. Belitsky, S. Hohenegger, G.P. Korchemsky, E. Sokatchev and A. Zhiboedov, Event shapes in $\mathcal{N}=4$ super-Yang-Mills theory, Nucl. Phys. B 884 (2014) 206 [arXiv: 1309.1424] [INSPIRE].

[69] A.V. Belitsky, S. Hohenegger, G.P. Korchemsky, E. Sokatchev and A. Zhiboedov, Energy-Energy Correlations in $N=4$ Supersymmetric Yang-Mills Theory, Phys. Rev. Lett. 112 (2014) 071601 [arXiv: 1311.6800] [INSPIRE].

[70] D.M. Hofman and J. Maldacena, Conformal collider physics: Energy and charge correlations, JHEP 05 (2008) 012 [arXiv:0803.1467] [INSPIRE].

[71] J.M. Maldacena, The large $N$ limit of superconformal field theories and supergravity, Int. J. Theor. Phys. 38 (1999) 1113 [hep-th/9711200] [INSPIRE].

[72] O. Gituliar and S. Moch, Fuchsia and Master Integrals for Energy-Energy Correlations at NLO in QCD, Acta Phys. Polon. B 48 (2017) 2355 [arXiv:1711.05549] [InSPIRE].

[73] L.G. Almeida, S.D. Ellis, C. Lee, G. Sterman, I. Sung and J.R. Walsh, Comparing and counting logs in direct and effective methods of QCD resummation, JHEP 04 (2014) 174 [arXiv: 1401.4460] [INSPIRE].

[74] C.W. Bauer, D. Pirjol and I.W. Stewart, Factorization and endpoint singularities in heavy to light decays, Phys. Rev. D 67 (2003) 071502 [hep-ph/0211069] [INSPIRE].

[75] J.C. Collins and D.E. Soper, Back-To-Back Jets: Fourier Transform from B to K-Transverse, Nucl. Phys. B 197 (1982) 446 [InSPIRE].

[76] J. Kodaira and L. Trentadue, Summing Soft Emission in QCD, Phys. Lett. B 112 (1982) 66 [INSPIRE].

[77] J. Kodaira and L. Trentadue, Single Logarithm Effects in electron-Positron Annihilation, Phys. Lett. B 123 (1983) 335 [INSPIRE].

[78] J.C. Collins, D.E. Soper and G.F. Sterman, Transverse Momentum Distribution in Drell-Yan Pair and $W$ and $Z$ Boson Production, Nucl. Phys. B 250 (1985) 199 [InSPIRE].

[79] H. Georgi and H.D. Politzer, Quark Decay Functions and Heavy Hadron Production in QCD, Nucl. Phys. B 136 (1978) 445 [inSPIRE].

[80] R.K. Ellis, H. Georgi, M. Machacek, H.D. Politzer and G.G. Ross, Perturbation Theory and the Parton Model in QCD, Nucl. Phys. B 152 (1979) 285 [InSPIRE].

[81] J.C. Collins and D.E. Soper, Parton Distribution and Decay Functions, Nucl. Phys. B 194 (1982) 445 [INSPIRE].

[82] J.C. Collins, D.E. Soper and G.F. Sterman, Factorization of Hard Processes in QCD, Adv. Ser. Direct. High Energy Phys. 5 (1989) 1 [hep-ph/0409313] [InSPIRE].

[83] J. Collins, Foundations of perturbative QCD, Cambridge University Press (2013) [INSPIRE].

[84] M.G. Echevarria, I. Scimemi and A. Vladimirov, Unpolarized Transverse Momentum Dependent Parton Distribution and Fragmentation Functions at next-to-next-to-leading order, JHEP 09 (2016) 004 [arXiv: 1604.07869] [INSPIRE]. 
[85] G.F. Sterman, Mass Divergences in Annihilation Processes. 1. Origin and Nature of Divergences in Cut Vacuum Polarization Diagrams, Phys. Rev. D 17 (1978) 2773 [inSPIRE].

[86] J.C. Collins and G.F. Sterman, Soft Partons in QCD, Nucl. Phys. B 185 (1981) 172 [INSPIRE].

[87] J.C. Collins, D.E. Soper and G.F. Sterman, Soft Gluons and Factorization, Nucl. Phys. B 308 (1988) 833 [INSPIRE].

[88] J. Chay, C. Kim, Y.G. Kim and J.-P. Lee, Soft Wilson lines in soft-collinear effective theory, Phys. Rev. D 71 (2005) 056001 [hep-ph/0412110] [INSPIRE].

[89] C.M. Arnesen, J. Kundu and I.W. Stewart, Constraint equations for heavy-to-light currents in SCET, Phys. Rev. D 72 (2005) 114002 [hep-ph/0508214] [INSPIRE].

[90] S. Fleming, A.K. Leibovich and T. Mehen, Resumming the color octet contribution to $e^{+} e^{-} \rightarrow J / \psi+X$, Phys. Rev. D 68 (2003) 094011 [hep-ph/0306139] [INSPIRE].

[91] I.W. Stewart, F.J. Tackmann and W.J. Waalewijn, Factorization at the LHC: From PDFs to Initial State Jets, Phys. Rev. D 81 (2010) 094035 [arXiv:0910.0467] [InSPIRE].

[92] I.W. Stewart, F.J. Tackmann and W.J. Waalewijn, The Quark Beam Function at NNLL, JHEP 09 (2010) 005 [arXiv: 1002.2213] [InSPIRE].

[93] R. Meng, F.I. Olness and D.E. Soper, Semiinclusive deeply inelastic scattering at small q(T), Phys. Rev. D 54 (1996) 1919 [hep-ph/9511311] [INSPIRE].

[94] X.-d. Ji, J.-p. Ma and F. Yuan, QCD factorization for semi-inclusive deep-inelastic scattering at low transverse momentum, Phys. Rev. D 71 (2005) 034005 [hep-ph/0404183] [INSPIRE].

[95] J.C. Collins, Proof of factorization for diffractive hard scattering, Phys. Rev. D 57 (1998) 3051 [Erratum ibid. D 61 (2000) 019902] [hep-ph/9709499] [INSPIRE].

[96] T. Gehrmann, T. Luebbert and L.L. Yang, Calculation of the transverse parton distribution functions at next-to-next-to-leading order, JHEP 06 (2014) 155 [arXiv:1403.6451] [inSPIRE].

[97] T. Gehrmann, T. Lubbert and L.L. Yang, Transverse parton distribution functions at next-to-next-to-leading order: the quark-to-quark case, Phys. Rev. Lett. 109 (2012) 242003 [arXiv:1209.0682] [INSPIRE].

[98] T. Lübbert, J. Oredsson and M. Stahlhofen, Rapidity renormalized TMD soft and beam functions at two loops, JHEP 03 (2016) 168 [arXiv: 1602.01829] [INSPIRE].

[99] M.G. Echevarria, I. Scimemi and A. Vladimirov, Universal transverse momentum dependent soft function at NNLO, Phys. Rev. D 93 (2016) 054004 [arXiv:1511.05590] [INSPIRE].

[100] M.-X. Luo, X. Wang, X. Xu, L.L. Yang, T.-Z. Yang and H.X. Zhu, TMD Beam Function and Fragmentation Function with Exponential Regulator, in preparation.

[101] G.P. Korchemsky and A.V. Radyushkin, Renormalization of the Wilson Loops Beyond the Leading Order, Nucl. Phys. B 283 (1987) 342 [InSPIRE].

[102] S. Moch, J.A.M. Vermaseren and A. Vogt, The Three loop splitting functions in QCD: The Nonsinglet case, Nucl. Phys. B 688 (2004) 101 [hep-ph/0403192] [INSPIRE].

[103] S. Moch, B. Ruijl, T. Ueda, J.A.M. Vermaseren and A. Vogt, Four-Loop Non-Singlet Splitting Functions in the Planar Limit and Beyond, JHEP 10 (2017) 041 [arXiv:1707.08315] [INSPIRE]. 
[104] P.A. Baikov, K.G. Chetyrkin, A.V. Smirnov, V.A. Smirnov and M. Steinhauser, Quark and gluon form factors to three loops, Phys. Rev. Lett. 102 (2009) 212002 [arXiv:0902.3519] [INSPIRE].

[105] R.N. Lee, A.V. Smirnov and V.A. Smirnov, Analytic Results for Massless Three-Loop Form Factors, JHEP 04 (2010) 020 [arXiv:1001.2887] [INSPIRE].

[106] T. Gehrmann, E.W.N. Glover, T. Huber, N. Ikizlerli and C. Studerus, Calculation of the quark and gluon form factors to three loops in QCD, JHEP 06 (2010) 094 [arXiv: 1004.3653] [INSPIRE].

[107] A. Vogt, S. Moch and J.A.M. Vermaseren, The Three-loop splitting functions in QCD: The Singlet case, Nucl. Phys. B 691 (2004) 129 [hep-ph/0404111] [InSPIRE].

[108] C.T.H. Davies and W.J. Stirling, Nonleading Corrections to the Drell-Yan Cross-Section at Small Transverse Momentum, Nucl. Phys. B 244 (1984) 337 [InSPIRE].

[109] C.T.H. Davies, B.R. Webber and W.J. Stirling, Drell-Yan Cross-Sections at Small Transverse Momentum, Nucl. Phys. B 256 (1985) 413 [INSPIRE].

[110] D. de Florian and M. Grazzini, Next-to-next-to-leading logarithmic corrections at small transverse momentum in hadronic collisions, Phys. Rev. Lett. 85 (2000) 4678 [hep-ph/0008152] [INSPIRE].

[111] Y. Li, A. von Manteuffel, R.M. Schabinger and H.X. Zhu, Soft-virtual corrections to Higgs production at $N^{3} L O$, Phys. Rev. D 91 (2015) 036008 [arXiv:1412.2771] [INSPIRE].

[112] L.J. Dixon, L. Magnea and G.F. Sterman, Universal structure of subleading infrared poles in gauge theory amplitudes, JHEP 08 (2008) 022 [arXiv:0805.3515] [INSPIRE].

[113] L. Freyhult, A. Rej and M. Staudacher, A Generalized Scaling Function for AdS/CFT, J. Stat. Mech. 0807 (2008) P07015 [arXiv:0712.2743] [INSPIRE].

[114] L. Freyhult and S. Zieme, The virtual scaling function of AdS/CFT, Phys. Rev. D 79 (2009) 105009 [arXiv:0901.2749] [INSPIRE].

[115] D. Fioravanti, P. Grinza and M. Rossi, Beyond cusp anomalous dimension from integrability, Phys. Lett. B 675 (2009) 137 [arXiv:0901.3161] [INSPIRE].

[116] L.J. Dixon, The Principle of Maximal Transcendentality and the Four-Loop Collinear Anomalous Dimension, JHEP 01 (2018) 075 [arXiv: 1712.07274] [INSPIRE].

[117] F. Cachazo, M. Spradlin and A. Volovich, Four-Loop Collinear Anomalous Dimension in $N=4$ Yang-Mills Theory, Phys. Rev. D 76 (2007) 106004 [arXiv:0707.1903] [InSPIRE].

[118] D. Kang, O.Z. Labun and C. Lee, Equality of hemisphere soft functions for $e^{+} e^{-}$, DIS and pp collisions at $\mathcal{O}\left(\alpha_{s}^{2}\right)$, Phys. Lett. B 748 (2015) 45 [arXiv: 1504.04006] [INSPIRE].

[119] I.Z. Rothstein and I.W. Stewart, An Effective Field Theory for Forward Scattering and Factorization Violation, JHEP 08 (2016) 025 [arXiv: 1601.04695] [INSPIRE].

[120] J.C. Collins and A. Metz, Universality of soft and collinear factors in hard-scattering factorization, Phys. Rev. Lett. 93 (2004) 252001 [hep-ph/0408249] [INSPIRE].

[121] J.C. Collins, Fragmentation of transversely polarized quarks probed in transverse momentum distributions, Nucl. Phys. B 396 (1993) 161 [hep-ph/9208213] [INSPIRE].

[122] J.C. Collins, Leading twist single transverse-spin asymmetries: Drell-Yan and deep inelastic scattering, Phys. Lett. B 536 (2002) 43 [hep-ph/0204004] [INSPIRE]. 
[123] S.J. Brodsky, D.S. Hwang and I. Schmidt, Initial state interactions and single spin asymmetries in Drell-Yan processes, Nucl. Phys. B 642 (2002) 344 [hep-ph/0206259] [INSPIRE].

[124] S.J. Brodsky, D.S. Hwang and I. Schmidt, Final state interactions and single spin asymmetries in semiinclusive deep inelastic scattering, Phys. Lett. B 530 (2002) 99 [hep-ph/0201296] [INSPIRE].

[125] A.V. Belitsky, X. Ji and F. Yuan, Final state interactions and gauge invariant parton distributions, Nucl. Phys. B 656 (2003) 165 [hep-ph/0208038] [INSPIRE].

[126] D. Boer, P.J. Mulders and F. Pijlman, Universality of T odd effects in single spin and azimuthal asymmetries, Nucl. Phys. B 667 (2003) 201 [hep-ph/0303034] [INSPIRE].

[127] C.J. Bomhof, P.J. Mulders and F. Pijlman, Gauge link structure in quark-quark correlators in hard processes, Phys. Lett. B 596 (2004) 277 [hep-ph/0406099] [INSPIRE].

[128] C.J. Bomhof, P.J. Mulders and F. Pijlman, The Construction of gauge-links in arbitrary hard processes, Eur. Phys. J. C 47 (2006) 147 [hep-ph/0601171] [INSPIRE].

[129] D.W. Sivers, Single Spin Production Asymmetries from the Hard Scattering of Point-Like Constituents, Phys. Rev. D 41 (1990) 83 [inSPIRE].

[130] M.D. Schwartz, K. Yan and H.X. Zhu, Collinear factorization violation and effective field theory, Phys. Rev. D 96 (2017) 056005 [arXiv:1703.08572] [inSPIRE].

[131] G.F. Sterman, Infrared divergences in perturbative QCD, AIP Conf. Proc. 74 (1981) 22.

[132] J.G.M. Gatheral, Exponentiation of Eikonal Cross-sections in Nonabelian Gauge Theories, Phys. Lett. B 133 (1983) 90 [INSPIRE].

[133] J. Frenkel and J.C. Taylor, Nonabelian eikonal exponentiation, Nucl. Phys. B 246 (1984) 231 [INSPIRE].

[134] P.F. Monni, E. Re and P. Torrielli, Higgs Transverse-Momentum Resummation in Direct Space, Phys. Rev. Lett. 116 (2016) 242001 [arXiv:1604.02191] [InSPIRE].

[135] M.A. Ebert and F.J. Tackmann, Resummation of Transverse Momentum Distributions in Distribution Space, JHEP 02 (2017) 110 [arXiv:1611.08610] [INSPIRE].

[136] D. Kang, C. Lee and V. Vaidya, A fast and accurate method for perturbative resummation of transverse momentum-dependent observables, JHEP 04 (2018) 149 [arXiv:1710.00078] [INSPIRE].

[137] J. Collins and T.C. Rogers, Connecting Different TMD Factorization Formalisms in QCD, Phys. Rev. D 96 (2017) 054011 [arXiv:1705.07167] [inSPIRE].

[138] T. Becher and G. Bell, Enhanced nonperturbative effects through the collinear anomaly, Phys. Rev. Lett. 112 (2014) 182002 [arXiv:1312.5327] [InSPIRE].

[139] P.B. Arnold and R.P. Kauffman, $W$ and $Z$ production at next-to-leading order: From large $q(t)$ to small, Nucl. Phys. B 349 (1991) 381 [INSPIRE].

[140] G.A. Ladinsky and C.P. Yuan, The Nonperturbative regime in QCD resummation for gauge boson production at hadron colliders, Phys. Rev. D 50 (1994) R4239 [hep-ph/9311341] [INSPIRE].

[141] Y. Makris, D. Neill and V. Vaidya, Probing Transverse-Momentum Dependent Evolution With Groomed Jets, JHEP 07 (2018) 167 [arXiv:1712.07653] [INSPIRE]. 
[142] M. Boglione, J. Collins, L. Gamberg, J.O. Gonzalez-Hernandez, T.C. Rogers and N. Sato, Kinematics of Current Region Fragmentation in Semi-Inclusive Deeply Inelastic Scattering, Phys. Lett. B 766 (2017) 245 [arXiv:1611.10329] [InSPIRE].

[143] K. Konishi, A. Ukawa and G. Veneziano, Jet Calculus: A Simple Algorithm for Resolving QCD Jets, Nucl. Phys. B 157 (1979) 45 [inSPIRE].

[144] A.J. Larkoski, G.P. Salam and J. Thaler, Energy Correlation Functions for Jet Substructure, JHEP 06 (2013) 108 [arXiv: 1305.0007] [INSPIRE].

[145] A.J. Larkoski, I. Moult and D. Neill, Power Counting to Better Jet Observables, JHEP 12 (2014) 009 [arXiv: 1409.6298] [INSPIRE].

[146] A.J. Larkoski, I. Moult and D. Neill, Building a Better Boosted Top Tagger, Phys. Rev. D 91 (2015) 034035 [arXiv:1411.0665] [INSPIRE].

[147] A.J. Larkoski, I. Moult and D. Neill, Analytic Boosted Boson Discrimination, JHEP 05 (2016) 117 [arXiv: 1507.03018] [INSPIRE].

[148] I. Moult, L. Necib and J. Thaler, New Angles on Energy Correlation Functions, JHEP 12 (2016) 153 [arXiv: 1609.07483] [InSPIRE].

[149] P.T. Komiske, E.M. Metodiev and J. Thaler, Energy flow polynomials: A complete linear basis for jet substructure, JHEP 04 (2018) 013 [arXiv:1712.07124] [INSPIRE].

[150] S.M. Freedman, Subleading Corrections To Thrust Using Effective Field Theory, arXiv: 1303.1558 [INSPIRE].

[151] I. Moult, L. Rothen, I.W. Stewart, F.J. Tackmann and H.X. Zhu, Subleading Power Corrections for N-Jettiness Subtractions, Phys. Rev. D 95 (2017) 074023 [arXiv: 1612.00450] [INSPIRE].

[152] R. Boughezal, X. Liu and F. Petriello, Power Corrections in the N-jettiness Subtraction Scheme, JHEP 03 (2017) 160 [arXiv: 1612.02911] [INSPIRE].

[153] I. Balitsky and A. Tarasov, Higher-twist corrections to gluon TMD factorization, JHEP 07 (2017) 095 [arXiv: 1706.01415] [INSPIRE].

[154] I. Moult, L. Rothen, I.W. Stewart, F.J. Tackmann and H.X. Zhu, $N$-jettiness subtractions for $g g \rightarrow H$ at subleading power, Phys. Rev. D 97 (2018) 014013 [arXiv:1710.03227] [INSPIRE].

[155] I. Balitsky and A. Tarasov, Power corrections to TMD factorization for Z-boson production, JHEP 05 (2018) 150 [arXiv:1712.09389] [INSPIRE].

[156] A.V. Manohar and I.W. Stewart, The Zero-Bin and Mode Factorization in Quantum Field Theory, Phys. Rev. D 76 (2007) 074002 [hep-ph/0605001] [INSPIRE].

[157] S. Catani and M.H. Seymour, The Dipole formalism for the calculation of QCD jet cross-sections at next-to-leading order, Phys. Lett. B 378 (1996) 287 [hep-ph/9602277] [INSPIRE].

[158] S. Catani and M.H. Seymour, A General algorithm for calculating jet cross-sections in NLO QCD, Nucl. Phys. B 485 (1997) 291 [Erratum ibid. B 510 (1998) 503] [hep-ph/9605323] [INSPIRE]. 